\title{
Versuche zum Nachweis von Cocosfett in Butterfett.
}

\author{
Von \\ C. Paal und Conrad Amberger.

\begin{abstract}
Mitteilung aus dem Pharmaceutisch-chemischen Institut der Univorsitat Erlangen.
\end{abstract}

In der vorstehenden Mittellung haben wir das Verhalten der flüchtigen Fettsäuren aus Butter und Cocosfett und aus Gemischen dieser beiden Fette, und zwar sowohl das der wasserlöslichen Säuren für sich, als auch des Gemenges der wasserlöslichen und der schwerlöslichen Säuren in Gestalt ihrer Alkalisalze gegen Zink- und Cadmiumsulfat unter verschiedenen Versuchsbedingungen untersucht.

Von der Tatsache ausgehend, daß die Menge der Glyceride der in Wasser schwerlöslichen, flüchtigen Fettsäuren beim Cocosfett erheblich größer ist wie beim Butterfett, dafo es daher für den Nachweis des ersteren Fettes in damit verfälschter Butter von Vorteil sein müsse, ein Destillationsverfahren anzuwenden, durch welches die schwerer flüchtigen, schwerlöslichen Säuren möglichst vollständig verflüchtigt werden, haben wir an Stelle des bei unseren bisherigen Versuchen benutzten, gewöhnlichen Destillationsverfahrens die Methode der Destillation im Wasserdampfistrome angewendet. Es lieb sich erwarten, dab hierbei nicht nur die im Cocosfett reichlicher wie im Butterfett vorhandenen Säuren, Capron-, Capryl- und Caprinsäure, möglichst vollständig in das Destillat übergehen, sondern daß auch noch etwas I Laurin- und Myristinsäure dadurch verflüchtigt würden. Da ferner buttersaures und capronsaures Cadminm in Wasser löslich sind und die nächst höheren Homologen schwer- bezw. unlösliche Cadmiumsalze bilden, so hofften wir auf dem angedeuteten Wege noch größere Differenzen zwischen den Mengen dieser Salze aus Butterfett einerseits, aus Cocosfett andererseits zu erhalten, um so einen sicheren Nachweis von Cocosfett in damit verfälschter Butter zu ermöglichen.

Aus der Zahl der dahinzielenden Versuche seien nur folgende angefüht:

I. $5 \mathrm{~g}$ Butterfett (reine Molker eibutter aus der Umgebung ErIangens) wurden in der üblichen Weise mit $10 \mathrm{cem}$ alkoholiseher Kalilange $(20 \mathrm{~g} \mathrm{Kalihydrat} \mathrm{in} 100 \mathrm{~cm} 70 \%$-igem Alkohol) verseift, die Seife in $100 \mathrm{ccm}$ ausgekochtem Wasser gelöst und mit $50 \mathrm{ccm}$ verdünnter Schwefelsäure (25 cem Schwefelsäure zu $1000 \mathrm{ccm}$ mit Wàsser verdünnt) die Fettsäuren in Freiheit gesetzt. Der die angesäuerte Seifenlösung enthaltende Kolben wurde dann einerseits mit dem Dampfentwickelungsapparat, andererseits mit einem absteigenden Kühler verbunden. Als Vorlage diente ein graduierter Mefscylmder. Wir destillierten in mäßigem Dampfstrom, bis $150 \mathrm{ccm}$ Destillat in die Vorlage übergegangen waren, wobei durch eine unter den Destillationskolben gestellte kleine Flamme das Elüssigkeitsniveau möglichst konstant gehalten wurde. Zur Überführung der im Kühler kondensierten schwerlöslichen Fettsäuren in die Vorlage wurde der Kühler mit einem Alkohol enthaltenden Kolben verbunden und $50 \mathrm{ccm}$ Alkohol in den Meßscylinder überdestilliert, wodurch die im Kühler haftenden Säuren vollständig gelöst wurden und in die Vorlage übergingen. Das durch ausgeschiedene Fettsäuren trübe, alkoholisch-wässerige Destillat versetzten wir hierauf nach Zugabe von etwas Phenolphthalein zur Überführung der Säuren in ihre Kalisalze mit einem gemessenen Überschußs von "10 N.-Kalilange und beseitigten dann den Alkaliüberschuß durch Zurücktitrieren mit ${ }^{1 / 10}$ N. Schwefelsäure bis eben zum Verschwinden der Phenolphthaleinfärbung. 
Aus der so exhaltenen Seifenlösung fällten wir nun direkt durch Zusatz von $2 \mathrm{ccm}$ einer 20\%-igen Cadmiumsulfatlosung die fettsauren Cadmiumsalze aus und brachten sie in der in der vorangehenden Mitteilung beschriebenen Art zur Wägang. In gleicher Weise bestimmten wir auch die Menge der Cadmiumsalze bei einer Mischung des gleichen Butterfettes mit $10 \%$ Cocosfett.

Da sich voraussehen ließ 3 , daß in den $150 \mathrm{~cm}$ Destillat aus reinem und cocosfetthaltigem Butterfett noch bei weitem nicht alle flüchtigen Fettsäuren enthalten sein konnten, so wurde die Destillation fortgesetzt und nochmals $150 \mathrm{ccm}$ Flüssigkeit überdestilliert; die im Kühler kondensierten Fettsäuren wurden durch Destillation von $50 \mathrm{ccm}$ Alkohol gelöst und so mit dem wässerigen Destillat vereinigt, darans die Cadmiumsalze gefällt und deren Menge bestimmt. Hierbei wurden folgende Ergcbnisse erhalten:

Gewicht der
$\begin{gathered}\text { Cadmiumfällung } \\ \text { (mg) }\end{gathered}$$\left\{\begin{array}{ccc}\text { aus der I. Fraktion } & \text { Reines Butterfett } & \begin{array}{c}\text { Dasselbe mit } \\ 10 \% \text { Cocosfett }\end{array} \\ \text { aus der II. Fraktion } & 73 & 119,5 \\ \hline \text { Zusammen } & 127 & 83,5 \\ \hline\end{array}\right.$

Der Unterschied in der Menge der Cadmiumsalze aus reiner und aus cocosfetthaltiger Butter ist somit sehr beträchtlich.

II. Bei weiteren Versuchen haben wir dann direkt $300 \mathrm{ccm}$ Flüssigkeit überdestilliert, die im Kühler haftenden Säuren durch Destillation von $50 \mathrm{~cm}$ Alkohol mil dem ersten Destillat vereinigt und darin die Cadmiumfalling bestimmt.

Nebon drei reinen Butterfetten wurden dann anch die entsprechenden, 10\% Cocosfett enthaltenden Gemische in der gleichen Art behandelt:

\begin{tabular}{|c|c|c|c|c|c|c|}
\hline & \multicolumn{2}{|c|}{$\begin{array}{c}\text { 1. Butter aus der Molkerei } \\
\text { Velgast (Prov. Branden- } \\
\text { burg) }{ }^{1} \text { ) }\end{array}$} & \multicolumn{2}{|c|}{$\begin{array}{l}\text { 2. Butter der Molkerei } \\
\text { Aschau (Oberbayern) }\end{array}$} & \multicolumn{2}{|c|}{$\begin{array}{l}\text { 3. Butter von einem Gat } \\
\text { am Radstädter Tauern } \\
\text { (1000 m hoch; Septbr. 1906) }\end{array}$} \\
\hline & $\begin{array}{l}\text { a) reines } \\
\text { Butterfett }\end{array}$ & $\begin{array}{l}\text { b) dasselbe mit } \\
10 \% \text { Cacosfett }\end{array}$ & $\begin{array}{l}\text { a) reines } \\
\text { Butterfett }\end{array}$ & $\begin{array}{l}\text { b) dasselbe mit } \\
10^{\circ} \text { o cocosfett }\end{array}$ & $\begin{array}{l}\text { a) reines } \\
\text { Butterfett }\end{array}$ & $\begin{array}{l}\text { b) dassslbe mit } \\
10 \% \text { Coeosfeit }\end{array}$ \\
\hline $\begin{array}{l}\text { Cadmium- } \\
\text { falllnng (mg) }\end{array}$ & 103 & 218 & 125 & 131 & 120 & 142 \\
\hline
\end{tabular}

Während in den Proben No. 1 die Menge der Cadmitumsalze aus dem Cocosfettgemisch mehr als das Doppelte von dem aus reinem Butterfett beträgt und un. gefähr mit dem Resultat des weiter oben beschriebenen Versuches (fraktionierte Destillation) übereinstimmt, sind diese Differenzen bei den beiden anderen Proben nur geringfügig, nämlich $6 \mathrm{mg}$ und $22 \mathrm{mg}$ Derartige große Unterschiede in dem Verhalten der reinen und der cocosfetthaltigen Proben, wie sie sich bei No. 1 b einerseits, bei No. 2 b und $3 \mathrm{~b}$ andererseits fanden, können nicht durch eine verschiedene Zusammensetzung der betreffenden Milchfette erklärt werden, sondern sie müssen auf andere Ursachen zurückgeführt werden. Eine derselben vermuteten wir in der Anwesenheit freier Schwefelsäure, von der Zerlegung der Seifen herrührend, weil wir bei einzelnen, in der vorhergehenden Mitteilung beschriebenen Versuchen die Erfahrung gemacht hatten, daß bei zunehmender Konzentration der freie Schwefelsäure und saures Kaliumsulfat enthaltenden Flüssigkeiten die Destillation insofern einen unregelmäßigen Verlauf nahm, als sich in der Menge der übergegangenen flüchtigen Fettsäuren, die als

1) Die Butter ist identisch mit No. 17 der Tabellen XI und XIV-XVI der vorstehenden Mitteilung. 
Schwermetallsalze bestimmt wurden, große Unterschiede zeigten, die nur entweder auf Überhitzung oder auf eine chemische Einwirkung der Schwefelsäure zurückgeführt werden konnten.

Um diese Wirkung unbeknnnter Art auszuschliefen, wurden bei den folgenden Versuchen die aus den Seifen durch verdünnte Schwefelsäure abgeschiedenen Fettsäuren nach dem Erkalten auf einer Siebfilterplatte abfiltriert, der Filterruckstand mit $50 \mathrm{~cm}$ Wasser in den Destillationskolben gebracht und nun wieder im Wasserdampfstrome destilliert. Ls wurden die Butterproben No. 2 a und $3 \mathrm{a}$ und die entsprechenden 10\% igen Cocosfettmischungen No. $2 \mathrm{~b}$ and $3 \mathrm{~b}$ der obigen Versuchsreihe verwendet, je $210 \mathrm{ccm}$ Flüssigkeit übergetrieben und die im Kühler kondensierten Fettsäuren durch Destillation von $40 \mathrm{ccm}$ Alkohol mit dem wässerigen Destillat vereinigt. Die Fällung der Cadmiumsalze gesehah in der oben angegebenen Weise. Bei diesen Versuchen gelangten nur die in Wasser schwerlöslichen, flüchtigen Säuren zur Bestimmung, da die löslichen in der angesäuerten Seifenlösung (Filtrat) ver. blieben waren; die Versuchsergebnisse waren folgende;

$\begin{array}{cccc}\text { No. 2a } & \text { No. 2b } & \text { No. 3a } & \text { No. 3 b } \\ \text { Butterfett } & \text { Dasselbe mit } & \text { Butterfett } & \begin{array}{c}\text { Dasselbe mit } \\ 10 \% \text { Cocosfett }\end{array} \\ 85 & 94 & 95 ; 112 & 124 ; 132\end{array}$

Cadmiumfällung (mg)

Wie ersichtlich, zeigen sich nur geringe Unterschiede in den Mengen der Cadmiumsalze
Ben Butterfetten im Vergleich zu den Cocosfettmischungen. Auch ergaben sich bei den aus den Butterfetten im Vergleich zu den Cocosfettmischungen. Auch ergaben sich bei den
Parallelversuchen der Proben No. $3 \mathrm{a}$ und $3 \mathrm{~b}$ unter sich beträcht]ich differierende Werte, obgleich hierbei ein etwaiger Einfluf der Schwefelsüure ausgeschlossen war.

Der Grund dieser Unregelmäfigkeiten ist nicht allein auf die bekannte Schwerflüchtigkeit der Fettsäuren von mittlerem Molekulargewicht, sondern auch auf die Schwierigkeit, die Destillation im Wasserdampfstrome bei allen Versuchen völlig gleichmäßig zu gestalten, zurückzuführen. Schon Orla Jensen ${ }^{1}$ ) hat darauf hingewiesen, daß die in Wasser schwer löslichen, flüchtigen Fettsäuren, die in den verflüssigten, hochmolekularen, nicht flüchtigen Säuren gelöst sind, sich mehr oder minder leicht verflüchtigen lassen, je nach der Oberflächengröße der flüssigen Fettsäureschicht und der Intensität des Siedens der wässerigen Flüssigkeit. Es werden also die Mengen der mit den Wasserdämpfen verflüchtigten, in Wasser schwerlöslichen Capryl- und Caprinsäure und besonders der noch schwerer flüchtigen Laurin- and Myristinsäure um so geringer sein, je ruhiger der Kolbeninhalt siedet und je weniger die geschmolzene Fettsäureschicht sich mit der siedenden Flüssigkeit emulgiert. Eine weit innigere Berührung und Mischung der Fettsäuren mit dem siedenden Wasser und dem Wasserdampf wie bei dem üblichen Destillationsverfahren nach ReichertMei liol findet bei der Destillation im Wasserdampfstrome statt, und dementsprechend ist auch die Menge der verflüchtigten, in Wasser schwer- bezw. unlöslichen, flüchtigen Säuren hierbei, wie unsere Versuche zeigen, recht beträchtlich, in Übereinstimmung mit der schon von Orla Jensen ausgesprochenen Ansicht, daß es durch Destillation im Wasserdampfstrome bei genügender Versuchsdauer möglieh sein müsse, die Gesamtmenge der im Cocosfett enthaltenen Capxyl- und Caprinsäure zu verflüchtigen.

Unsere Versuche, von denen, wie erwähnt, nur wenige vorstehend angeführt wurden, lehren aber, daß man auf diesen Wege sowohl bei Parallelversuchen mit

1) Diese Zeitschrift, 1905, 10, 265. 
einer und derselben Fettprobe wie auch bei verschiedenen Proben von ähnlicher $\mathrm{Zu}$ sammensetzung und bei relativ kurzer Destillationsdauer keine übereinstimmenden Ergebnisse bekommt, weil es praktisch unmöglich ist, die zu destillierende Flüssigkeit während der Destillation stets auf gleichem Niveau und vor allem die Dampfzufubr und den Dampfdruck auch nur annähernd konstant zu erhalten. Auch der Wärmeverlust durch Ausstrahlung ist je nach der AuBentemperatur verschieden.

Alle diese UnregelmäBigkeiten mußten sich aber fast vollständig vermeiden lassen, wenn es gelang, die Destillation im Dampfstrome innerhalb des Dampfentwicklers selbst auszuführen. Zu diesem Zwecke wurde von dem einen von uns (A mberger) der nebenstehend abgebildete Apparat (Fig. 1) konstruiert, bei welchem sich die Destillation im Wasserdampfstrome innerhalb des Kolbens vollzieht, in dem der Dampf erzeugt wird.

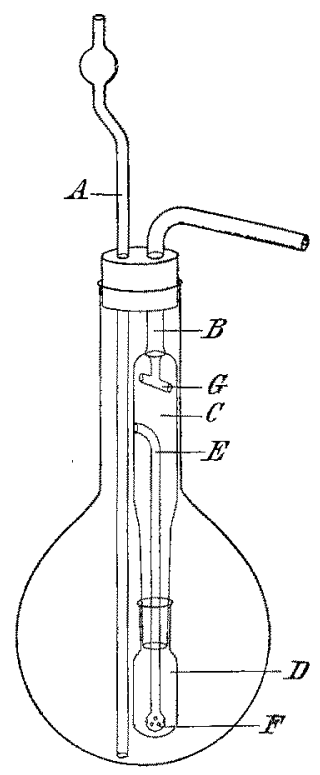

Fig. 1.

Der Apparat setzt sich zusammen aus einem weithalsigen Rundkolben von ungefähr $1 \frac{1}{2}$ Liter Inhalt, in welchem der Wasserdampf entwickelt wird. Der Kolben ist durch einen doppelt durcbbohrten Kautschukstopfen verschlossen, durch dessen eine Bohrung ein Steigerohr $\mathbf{A}$, durch die andere der Hals B des eigentlichen Destillationsapparates gesteckt wird. Dieser besteht aus zwei Teilen, einem sich vom Hals nach abwärts erweiternden Rohr C, über dessen unteres Ende ein eingeschliffenes Gläschen $\mathrm{D}$ geschoben werden kann in welches die mit Wasserdampf zu destillierende Substanz gebracht wird. Der im Kolben entwickelte Wasserdampf tritt durch das an das Destillationsrohr $\mathrm{C}$ angeschmolzene enge Glasrohr $\mathrm{E}$ ein und strömt durch dessen untere, kugelförmige, durchlöcherte Erweiterung $F$ in das Gläschen D ein Im Halse $B$ ist zur Sicherung gegen mechanisches Mitreißen von Wasser- und Fettsäuretröpfchen ein Tropfenfänger $G$ angebracht. Das aus dem Kolben ragende Ende des Halses B ist knieförmig nach abwärts gebogen und wird mit dem in der Zeichnung weggelassenen Kühler verbunden ${ }^{\mathbf{1}}$ ).

Versuchs-Ausführung: $2,5 \mathrm{~g}^{2}$ ) des zu untersuchenden Fettes, genau abgew ogen, werden mit $10 \mathrm{~g}$ einer Glycerin-Natronlauge verseift, die durch Auffüllen von $20 \mathrm{ccm}$ einer 50\%-igen Natronlauge mit Glycerin auf $200 \mathrm{ccm}$ erhalten wird (Leffmann und Beam'sche Lauge). Die Seife löst man in $50 \mathrm{ccm}$ heißem ausgekochtem Wasser und scheidet aus der auf $55-60^{\circ}$ abgekühlten Lösung die freien Fettsäuren durch $25 \mathrm{ccm}$ verdünnte Schwefelsäure $(25 \mathrm{ccm}$ Schwefelsäure im Liter) ab. Nach ungefähr 10-12-stündigem Stehen wurde das erstarrte Fettsäuregemisch auf einem mit einer Porzellanfilterplatte versehenen kleinen Trichter ohne oder unter sehr gelinder Anwendung der Saugpumpe abfiltriert und mit $50 \mathrm{ccm}$ kalten Wassers nach-

1) Der Apparat ist als D.R.G.M. angemeldet worden und kann von der Firma Franz Huge $\mathbf{r}$ sh off in Leipzig (Carolinenstrake) bezogen werden.

2) Während bei den oben erwähnten Versuchen, die flüchtigen Fettsäuren aus Butterfett und Cocosfett im Wasserdampfstrome überzutreiben, von je $5 \mathrm{~g}$ Fett ausgegangen wurde, genügt bei Anwendung des vorstenend beschriebenen Apparates die Hälfte dieser Fettsaenge. 
gewaschen. Die Porzellanfilterplatte war vor der Filtration mit einer genau passenden Scheibe Filtrierpapier bedeckt worden. Die darauf befindlichen Fettsäuren wurden samt der Papierscheibe möglichst verlustlos in das Gläschen D des Apparats gegeben und etwa noch am Trichter haftende Fettsäurepartikelchen durch Nachspülen mit $5 \mathrm{ccm}$ einer $1 \%$-igen Schwefelsäure ebenfalls in das Gläschen gebracht, welches dann an das Destillationrohr $\mathrm{C}$ angesetzt wurde. Der so beschickte Destillationsapparat wird dann in den $800 \mathrm{ccm}$ Wasser und $1 \mathrm{~g}$ fein gepulverten Bimsstein enthaltenden Dampfentwickelungskolben so eingefügt, daß sich der Boden des die Fettsäuren enthaltenden Gläschens ungefähr $1 \mathrm{~cm}$ über dem Boden des Rundkolbens befindet, worauf der aus dem Kolben herausragende, knieförmig gebogene Teil des Halses B mit dem schräg nach abwärts gerichteten Kühler verbunden wird, welcher in einem als Vorlage dienenden, graduierten Meßcylinder von $250 \mathrm{ccm}$ Inhalt mündet.

Man erhitzt dann den Kolben; der sich auf einem Drahtnetz befindet, mittels eines Pilzbrenners und leitet die Destillation so, dab innerhalb $35-40$ Minuten $200 \mathrm{ccm}$ Destillat übergehen. Sind diese überdestilliert, so unterbricht man sofort die Destillation, entfernt den Destillierapparat und verbindet an dessen Stelle einen mit neutralisiertem absolutem Alkohol beschickten Kolben mit dem Kühler Durch Destillation von genau $50 \mathrm{ccm}$ Alkohol werden die im Kühler haftenden Fettsäuren gelöst und vereinigen sich mit dem wässerigen Destillat. Das nun $250 \mathrm{ccm}$ betragende, alkoholisch-wässerige Destillat, eine durch darin suspendierte, schwer lösliche Fettsäuren getrübte Flüssigkeit, wird dann unter Zusatz von etwas Phenolphthalein mit 1/10 N.-Kalilauge deutlich alkalisch gemacht, wozu je nach der Menge der flüchtigen Säuren 10-15 ccm erforderlich sind. Nach kräftigem Schütteln titriert man die nun klare, die Säuren als Kaliumsalze enthaltende Lösung mit 1/10 N.-Schwefelsäure bis eben zum Verschwinden der Phenolphthaleinfärbung zurück und erfährt so die zur Neutralisation der flüchtigen, in Wasser schwer löslichen Fettsäuren erforderliche Menge 1/10 N.-Kalilauge. Um bei dem darauffolgenden Konzentrieren des neutralisierten Destillates eine Verflüchtigung von Fettsäuren infolge von Hydrolyse möglichst hintanzuhalten, wird noch $1 \mathrm{ccm} 1 / 10$ N.-Kalilauge zugegeben, die nun alkalische Lösung in einer Porzellanschale auf dem Wasserbade auf etwa $40-45 \mathrm{ccm}$ eingeengt, ohne Verlust in einen kleinen graduierten Meßcylinder gebracht, mit destilliertem Wasser auf $50 \mathrm{ccm}$ aufgefüllt und mit 1/10 N.-Schwefelsäure bis eben zum Verschwinden der Phenolphthaleinfärbung neutralisiert. Ein Säureüberschuß ist sorgfältig zu vermeiden. Durch Zugabe von $2 \mathrm{ccm}$ einer $20^{\circ}, 0$-igen Cadmiumsulfatlösung (bei Anwendung von Cocosfett oder mehr als $40 \%$ Cocosfett enthaltendem Butterfett sind $4 \mathrm{ccm}$ Cadmiumsulfatlösung erforderlich) führt man das in der Lösung vorhandene Gemisch der fettsauren Kalisalze in die Cadmiumsalze über, von denen sich die schwerlöslichen als weißer, voluminöser Niederschlag ausscheiden, der sich nach mehrmaligem Umschwenken des Cylinders gut absetzt und nach ungefähr einstündigem Stehen im Gooch-Tiegel abfiltriert wird, wobei das Filtrat zum Nachspülen des an den Wänden des Cylinders hängenden .Niederschlages benutzt wird. Die im Tiegel angesammelte Fällung lockert man dann mit einem dünnen Glasstab etwas auf und wäscht in der Weise aus, dah immer erst dann neues Waschwasser zugegeben wird, wenn das vorhergehende völlig abgelaufen ist. So gelingt es, mit $50 \mathrm{ccm}$ Waschwasser den Niederschlag sulfatfrei zu erhalten.

Die Fällung wird im Tiegel bis zur Gewichtskonstanz bei $102-106^{\circ}$ getrocknet, 
wozu gewöhnlich einstündiges Erhitzen genügt, nach dem Erkalten gewogen und so die Menge der schwer- bezw. unlöslichen Cadmiumsalze ermittelt.

Beim Trocknen schmelzen nach einiger Zeit die Cadmiumsalze, und der anfangs rein weiße Niederschlag geht dann nach dem Erkalten in eine mehr oder minder bräunlich gefärbte, hornartige Masse über, Aus unseren, in der vorstehenden Mitteilung beschriebenen. Versuchen geht jedoch hervor, daf bierbei weder eine Zersetzung, noch eine Verflüchtigung von Fettsäuren, etwa in Form ihrer Anhydride, in merklicher Menge stattfindet.

\section{Versuche mit reinem Butterfett, Cocosfett und Mischungen der beiden Fette.}

Unter genauer Befolgung der vorstehend gegebenen Vorschrift wurden die Cadmiumsalzwerte erbalten, wie sie in den folgenden Tabellen I, II und III (S. 30-32) zusammengestellt sind. Die Mengen der Cadmiumsalze sind in Miligrammen angegeben; wir bezeichnen diese Werte der Kürze halber als „CadmiumZahlen".

In der Tabelle $I$ sind die Versuchsergebnisse von einer Reihe reiner, sogenannter „normalers Butterfette, deren Juckenack-Pasternack'sche Differenzen nur wenig von \pm 0 abweichen, zusammengestellt. Die Proben sind geordnet nach aufsteigenden Reichert-Meißl'schen Zahlen und innerhalb dieser nach aufsteigenden Verseifungszahlen ${ }^{1}$ ).

Zur weiteren Charakterisierung sind ferner Herkunft, Fütterung, soweit deren Art und Menge ermittelt werden konnte, und die Zeit der Probenentnahme angeführt. In dieser Tabelle $I$ sind neben den Cadmium-Zahlen der reinen Butterfette auch die der betreffenden Mischungen mit je 10\% Cocosfett verzeichnet. Die 32 untersuchten reinen Butterproben stammten teils von Molkereien, teils von größeren und kleineren Landgütern, teils auch wurden sie gelegentlich der von uns veranlaßten Fütterungversuche an Küben in Altdorf bei Nürnberg aus der Milch der mit Malzkeimen, Cocoskuchen und Rüben gefütterten Tiere im Laboratorium gewonnen. In die Tabellen I und III sind nur solche Butterproben aufgenommen worden, die relativ kleine Juckenack-Pasternack'sche Differenzen zeigen. Über jene Butterfette, welche nach längerer Fütterung mit den erwähnten Futtermitteln erhalten wurden und in ihrer Zusammensetzung von der Norm stark abweichende Werte ergaben, wird weiter unten berichtet.

Tabelle II enthält die Cadmium-Zahlen nebst den übrigen Konstanten von 3 Cocosfetten verschiedener Herkunft, wie wir sie im Handel erhielten oder sie uns aus Cocosnüssen durch Extraktion mit Äther selbst darstellten.

In Tabelle III sind die Cadmium-Zahlen angeführt, welche wir bei Untersuchung von 3 in Tabelle $I$ angegebenen reinen Butterfetten erhielten, die mit je 10, 15, 20 und $30 \%$ Palmin zusammengeschmolzen waren.

Die Cadmium-Zahlen sämtlicher in der. Tabelle I angeführten Butterproben bewegen sich, obwohl die reinen Fette hinsichtlich ibrer Reichert-MeiB1- und Verseifungszahlen ein recht verschiedenes Analysenbild zeigen, doch nur zwischen 70 und $90 \mathrm{mg}$, also innerhalb der engen Grenze von $20 \mathrm{mg}$ Differenz. Ebenso verhalten sich die bei den 10\% Cocosfett enthaltenden Butterfetten gefundenen Cad-

1) Die in den Tabellen angeführten Reichert-Meifl'schen Zahlen und Verseifungazahlen sind Mittelwerte aus $2-3$ Bestimmungen. 
mium-Zahlen, die zwischen 104 und $124 \mathrm{mg}$ liegen, demnach ebenfalls, wie bei den reinen Butterfetten, zwischen dem niedrigsten und böchsten Wert eine Differenz von $20 \mathrm{mg}$ ergeben.

Einem Gehalt an 10\% Cocosfett in den Proben der Tabelle I entspricht den reinen Butterproben gegenüber eine Zunahme der Cadmium-Zablen um durchschnittlich $33 \mathrm{mg}$ (niedrigster Wert $28 \mathrm{mg}$ bei No. 6, höchster Wert $38 \mathrm{mg}$ bei No. 19 u. 20). Im Gegensatz zu den eingangs angeführten Versuchen liefert demnach die Destillation im Wasserdampfstrome in dem vorstehend beschriebenen Apparate sehr gleiehmäßige Ergebnisse. Bei allen untersuchten Butterfetten steigt, wie aus der Tabelle ersichtlich, die Cadmium-Zahl nicht über 90 (No. 28 und 32), während die niedrigsten Cadmium-Zahlen für die 10\% Cocosfett enthaltenden Proben bei 104, 106 und 108 liegen (No. 1, 2, 7); ihnen entsprechen bei den reinen Butterfetten ebenfalls die niedrigsten gefundenen Werte, 70, 71, 70,4 (No. 1, 2, 7), so daß also zwischen der höchsten Cadmium-Zahl von reiner Butter und der niedrigsten von cocosfetthaltigen Proben eine Differenz von $14 \mathrm{mg}$ liegt.

Aus Tabelle II, die Cadmium-Zahlen von Cocosfetten verschiedener Herkunft enthaltend, ergibt sich das Verbältnis der Cadmium-Zahlen dieses Fettes zu dem der reinen Butterfette. Die Cadmium-Zahlen des Cocosfettes liegen zwischen 441 und 470 , jene der Butterfette zwischen 70 und 90 ; sie stehen also umgefähr im Verhältnis von $5,5: 1$.

In der Tabelle III sind die Ergebnisse der Untersuchung von drei Butterfetten der Tabelle I (No. 5, 7, 32), erstere beiden mit niedrigen Reichert-M eibl'schen Zablen und Verseifungszahlen, die dritte mit hoher Reichert-Meißl'scher Zahl und Verseifungszahl angefüht. Diese Fette wurden mit steigenden Mengen Cocosfett vermischt und die Cadmium-Zahlen dieser Mischungen bestimmt. Die Werte steigen mit zunehmendem Cocosfettgehalt regelmäßig und beträchtlich an, sodaß z. B. die Cadmium-Zahlen der mit $20 \%$ Cocosfett vermischten Butterfette bereits doppelt so hoch sind, wie die der zugehörigen reinen Butterfette. Neben den Cadmium-Zahlen sind in den drei Tabeilen auch Titrationswerte angeführt. Es ist darunter die Anzahl cem 1/10 N.- Kalilauge zu verstehen, die zur Neutralisation der schwerlöslichen ${ }^{1}$ ) flüchtigen Fettsäuren im Destillat erforderlich sind.

Diese Zahlen haben nur orientierende Bedeutung insofern, als man annehmen kann, daks Butterfette, deren Titrationswert unter 8 liegt, nicht mit erheblichen Mengen Cocosfett verfä].scht sind. Bei geringen Cocosfettzusätzen lässt das Verfahren jedoch im Stich, wie aus den Versuchen No. 10, 13 und 19 der Tabelle I hervorgeht, deren Titrationswerte für die $10 \%$ Cocosfett enthaltenden Proben unter 8 liegen. Wir erwarteten übrigens auch nieht mit Hilfe der Titration der flüchtigen Säuren einen Zusatz von wenig Cocosfett in Butter nachweisen za kömnen.

Die Tritationswerte sind deshalb von einigem Interesse, weil sie im Vergleich mit den Cadmium-Zahlen die Überlegenheit der Methode zeigen, durch Fällung mit gewissen Schwermetallsalzen gerade jene flüchtigen Fettsäuren abseheiden und bestimmen zu können, die in überwiegender Menge im Cocosfett, in relativ geringer Menge dagegen im Butterfett in Form der Glyceride vorhanden sind. Hierzu sind, wie auch schon aus den in der vorstehenden Mitteilung angegebenen Versuchen hervorgeht, $\mathrm{Cadmium-und} \mathrm{Zinksulfat} \mathrm{besonders}$ geeignet.

[Fortsetzung S. 32.]

1) Die löslichen flüchtigen Säuren sind vorher durch Filtration entfernt worden. 
Tabelle I. Reine nor-

\begin{tabular}{|c|c|c|}
\hline No. & Herkunft der Butter & Art der Fütterung \\
\hline $\begin{array}{l}1 \\
2 \\
3 \\
4\end{array}$ & 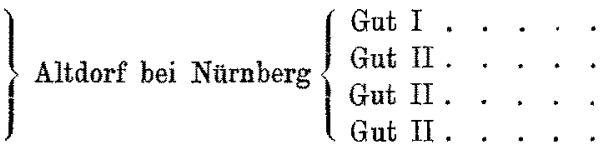 & $\begin{array}{c}\text { Malzkeimfütterung } \\
\text { Heu, Häcksel, } 15 \text { Pfd. Rüben } \\
\text { Heu, Häcksel, } 2 \text { Pfd. Cocoskuchen } \\
\text { Gras, Häcksel }\end{array}$ \\
\hline 5 & 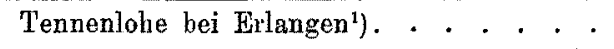 & Gras, Heu \\
\hline 6 & $\begin{array}{c}\text { Radstädter Tauern (Salzburg). Hoch-Alpe. } \\
\text { Höhe } 1600 \mathrm{~m} \text {. }\end{array}$ & Weidegang \\
\hline $\begin{array}{l}7 \\
8 \\
9\end{array}$ & Altdorf bei Nürnberg $\left\{\begin{array}{lllll}\left.\text { Gut II }{ }^{2}\right) & . & . & . & . \\
\text { Gut II } . & . & . & \cdot & . \\
\text { Gut II } . & . & . & . & .\end{array}\right.$ & $\begin{array}{c}\text { Heu, Gras } \\
\text { Heu, Gras, Häcksel, } 1^{1 / 2} \text { Pfd. Cocoskuchen } \\
\text { Heu, Häcksel, } 20 \text { Pfd. Rüben }\end{array}$ \\
\hline 10 & Braunau (Oberösterreich) . . . . . . . & unbekannt \\
\hline 11 & Altdorf bei Nürnberg, Gut II. . . . . . & Heu, Häcksel, 25 Pfd. Rüben \\
\hline 12 & Molkerei Großenbuch (Oberfranken) . . . & Heu, Gras \\
\hline 13 & Windsbach (Mittelfranken) . . . . . . & Weidegang \\
\hline 14 & Altdorf bei Nürnberg, Gut II. . . . . . & Heu, Gras, $1^{1 / 2}$ Pfd. Cocuskuchen \\
\hline 15 & Gräfenberg (Oberfranken) . . . . . . . & unbekannt \\
\hline $\begin{array}{l}16 \\
17 \\
18\end{array}$ & Altdorf bei Nürnberg $\left\{\begin{array}{lllll}\text { Gut II } & . & . & . & . \\
\text { Gut II } & . & \cdot & . & \cdot \\
\text { Gut II } & . & \cdot & . & \cdot\end{array}\right.$ & $\begin{array}{l}\text { Weidegang } \\
\text { Gras, 30 Pfd. Rüben } \\
\text { Treber } 24 \text { Pfd., Häcksel }\end{array}$ \\
\hline 19 & Braunau (Oberösterreich) & anbekannt \\
\hline 20 & Altdorf bei Nürnberg, Gut II . . . . . . & 40 Pfd. Rüben \\
\hline 21 & Molkerei Schwiebus (Provinz Brandenburg) & unbekannt \\
\hline 22 & Molkerei Gobsan (Provinz Brandenburg) & Grünfutter, Rüben, Schlempe \\
\hline 23 & Reichelsdorf bei Nürnberg. . . . . . . & Weidegang \\
\hline 24 & Molkerei Grofenbuch (Oberfranken) . . . & Weidegang \\
\hline 25 & Kochstedt bei Dessau . . . . & unbekannt \\
\hline 26 & Molkerei Sagast (Provinz Brandenburg) & Klee, Heu, Haferstroh, Weidegang \\
\hline 27 & Fichtelberg (Oberfranken) . . . . . . . & unbekannit \\
\hline $28 \mid$ & Molkerei Bruck (Provinz Brandenburg) . & Grünfutter, Heu, Rübenblätter, Cocoskuchen| \\
\hline 29 & $\begin{array}{c}\text { Radstädter Tauern (Salzburg), Gut I, Höhe } \\
1000 \mathrm{~m}\end{array}$ & Heu, Wruken \\
\hline 30 & Klentzsch bei Dessau . . . . . . . . & Rüben, Kartoffeln \\
\hline $\begin{array}{l}31 \\
32\end{array}$ & $\left\{\begin{array}{c}\text { Radstädter Tauern (Salzburg), Kah Isidora } \\
\text { Gat II, Höhe } 900 \mathrm{~m}\end{array}\right.$ Kuh Sarah. & $\begin{array}{l}\text { Heu, Gras } \\
\text { Heu }\end{array}$ \\
\hline
\end{tabular}

1) Kuh ist altmilchend, 28 Wochen trächtig.

2) Kuh ist altmilchend, 
male Butterfette.

\begin{tabular}{|c|c|c|c|c|c|c|c|}
\hline \multirow{2}{*}{$\begin{array}{c}\text { Zeit der } \\
\text { Probenentnalume }\end{array}$} & \multirow{2}{*}{$\left|\begin{array}{c}\text { Roiehert- } \\
\text { Meisisgehe } \\
\text { Zahl }\end{array}\right|$} & \multirow{2}{*}{$\begin{array}{c}\text { Versiffungs- } \\
\text { zalil }\end{array}$} & \multirow{2}{*}{\begin{tabular}{|c|} 
Jucke- \\
nack-Pa- \\
aternaek- \\
sche \\
Differenz
\end{tabular}} & \multicolumn{2}{|c|}{$\begin{array}{c}\text { Titration aer đutuchtigen } \\
\text { Săuren }\end{array}$} & \multicolumn{2}{|c|}{ 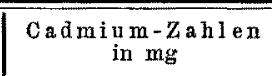 } \\
\hline & & & & $\mid \begin{array}{l}\text { der reinen } \\
\text { Butterfette }\end{array}$ & $\begin{array}{c}\text { der Butter } \\
\text { fette mit } \\
10 \% / 0 \text { Cocos- } \\
\text { fett }\end{array}$ & $\begin{array}{l}\text { der reinen } \\
\text { Butterfette }\end{array}$ & $\begin{array}{l}\text { der Butter- } \\
\text { fette mit } \\
10 \% \text { Coeog- } \\
\text { fett }\end{array}$ \\
\hline April 1907 & 18,0 & 218,0 & \pm 0 & 6,8 & 8,6 & 70,0 & 104,0 \\
\hline Oktober 1907 & 22,4 & 219,2 & $+3,2$ & 7,3 & 9,0 & 71,0 & 106,0 \\
\hline Oktober 1907 & 22,7 & 221,0 & $+1,7$ & 7,9 & - & 76,4 & 112,2 \\
\hline Oktober 1907 & 22,3 & 221,4 & $+0,9$ & 7,4 & - & 83,0 & 114,2 \\
\hline Mai 1907 & 22,2 & 224,2 & $-2,0$ & 8,4 & 10,5 & 82,0 & 116,0 \\
\hline September 1906 & 22,4 & 224,5 & $-2,1$ & 7,9 & - & 86,4 & 114,4 \\
\hline Oktober 1907 & 23,3 & 222,7 & $+0,6$ & 8,6 & 9,2 & 70,4 & 108,0 \\
\hline Oktober 1907 & 23,5 & 221,9 & $+1,6$ & 8,0 & - & 74,0 & 116,0 \\
\hline Oktober 1907 & 23,4 & 221,8 & $+1,6$ & - & - & 79,8 & 116,8 \\
\hline Oktober 1906 & 24,9 & 223,1 & $+1,8$ & $5, \overline{9}$ & 7,5 & 83,0 & 117,0 \\
\hline Oktober 1907 & 24,9 & 223,6 & $+1,3$ & 8,4 & 9,8 & 77,0 & 110,0 \\
\hline Mai 1907 & 24,2 & 224,5 & $-0,3$ & 8,2 & 8,8 & 82,0 & 119,0 \\
\hline September 1906 & 24,0 & 227,0 & $-3,0$ & 6,2 & 7,3 & 84,0 & 118,0 \\
\hline Juli 1907 & 25,1 & 228,8 & $+1,3$ & 7,7 & 8,9 & 81,4 & 114,0 \\
\hline Mai 1907 & 25,6 & 225,3 & $+0,3$ & 7,7 & 9,6 & 78,0 & 115,0 \\
\hline Juli 1907 & 25,6 & 226,5 & $-0,9$ & 8,5 & 9,7 & 82,0 & 116,0 \\
\hline Oktober 1907 & 25,5 & 227,6 & $-2,1$ & 8,0 & 11,5 & 81,0 & 116,0 \\
\hline März 1907 & 26,2 & 224,5 & $+1,7$ & 7,9 & 11,0 & 88,0 & 122,6 \\
\hline Oktober 1906 & 26,6 & 228,1 & $-1,5$ & 6,6 & 7,9 & 86,0 & 124,0 \\
\hline Oktober 1907 & 26,1 & 228,6 & $-2,5$ & 9,2 & 10,5 & 86,0 & 124,0 \\
\hline November 1906 & 26,6 & 229,0 & $-2,4$ & 7,5 & 9,3 & 77,0 & 114,0 \\
\hline November 1906 & 27,1 & 224,0 & $+3,1$ & 7,6 & 8,6 & 78,8 & 116,6 \\
\hline Oktober 1906 & 27,7 & 227,2 & $+0,5$ & 7,3 & 8,6 & 84,0 & 119,8 \\
\hline Oktober 1906 & 27,5 & 228,3 & $-0,8$ & 6,2 & 8,1 & 81,6 & 118,4 \\
\hline Januar 1907 & 27,7 & 228,6 & $-0,9$ & 7,4 & 8,6 & 80,0 & 115,0 \\
\hline November 1906 & 28,2 & 224,0 & $+4,2$ & 7,5 & 9,1 & 79,6 & 114,0 \\
\hline Oktober 1907 & 28,6 & 226,5 & $+2,1$ & 7,4 & - & 79,0 & 112,2 \\
\hline Dezember 1906 & 28,4 & 230,5 & $-2,1$ & - & - & 90,0 & 122,0 \\
\hline April 1907 & 29,0 & 227,8 & $+1,2$ & 8,6 & 10,1 & 87,4 & 122,6 \\
\hline Oktober 1906 & 29,0 & 231,3 & $-2,8$ & 8,5 & 9,9 & 89,0 & 124,0 \\
\hline $\begin{array}{l}\text { Mai } 1907 \\
\text { April } 1907\end{array}$ & $\begin{array}{l}31,0 \\
32,3\end{array}$ & $\begin{array}{l}229,8 \\
230,6\end{array}$ & $\begin{array}{l}+1,2 \\
+1.7\end{array}$ & $\begin{array}{l}8,8 \\
8,6\end{array}$ & $\begin{array}{l}11,7 \\
10,4\end{array}$ & $\begin{array}{l}88,0 \\
90,0\end{array}$ & $\begin{array}{l}121,6 \\
121,4\end{array}$ \\
\hline
\end{tabular}

32 Wochen trächtig. 
Tabelle II. Cocosfett.

\begin{tabular}{c|c|c|c|c|c|c}
\hline No. & Herkunft & $\begin{array}{c}\text { Reichert- } \\
\text { Meißsl'sche } \\
\text { Zahl }\end{array}$ & $\begin{array}{c}\text { Versei- } \\
\text { fungs-Lahl }\end{array}$ & $\begin{array}{c}\text { Jukkenack- } \\
\text { Pasternack- } \\
\text { sehe Differenz }\end{array}$ & $\begin{array}{c}\text { Titration } \\
\text { der flíchti- } \\
\text { gen Säuren }\end{array}$ & $\begin{array}{c}\text { Cadmium- } \\
\text { Zahl } \\
\text { mg }\end{array}$ \\
\hline 1 & $\begin{array}{c}\text { Cocosfett, aus Nüssen ex- } \\
\text { trahiert }\end{array}$ & 8,3 & 259,4 & $-51,1$ & 30,7 & $\mathbf{4 7 0}$ \\
\hline 2 & Palmin des Handels & 7,8 & 257,6 & $-49,8$ & 27,8 & $\mathbf{4 5 5}$ \\
\hline 3 & $\begin{array}{c}\text { Albin des Handels (Mann- } \\
\text { heim) }\end{array}$ & 7.5 & 258,5 & $-51,0$ & 27,2 & $\mathbf{4 4 1}$ \\
\hline
\end{tabular}

Tabelle III. Mischungen von Butterfett mit Cocosfett ${ }^{1}$ ).

\begin{tabular}{|c|c|c|c|c|c|c|c|c|c|c|c|c|}
\hline \multirow[b]{2}{*}{ Herkunft } & \multicolumn{4}{|c|}{ Reine Butterfette } & \multicolumn{2}{|c|}{$\begin{array}{l}\text { Butterfett mit } \\
10^{\circ}{ }_{0} \text { Cocosfett }\end{array}$} & \multicolumn{2}{|c|}{$\begin{array}{l}\text { Butterfett mit } \\
15 \% \text { Cocosfett }\end{array}$} & \multicolumn{2}{|c|}{$\begin{array}{l}\text { Butterfott mit } \\
20 \% \text { Cocosfett }\end{array}$} & \multicolumn{2}{|c|}{$\begin{array}{l}\text { Butterfett mit } \\
30 \% \text { Cocosfett }\end{array}$} \\
\hline &  & $\begin{array}{l}\text { Tersei- } \\
\text { fungs- } \\
\text { ZahI }\end{array}$ & $\begin{array}{c}\text { Titra- } \\
\text { tion }\end{array}$ & $\begin{array}{c}\text { Cad- } \\
\text { mium- } \\
\text { Zahl } \\
\text { mg }\end{array}$ & $\begin{array}{l}\text { Titra- } \\
\text { tion }\end{array}$ & $\begin{array}{c}\text { Oad- } \\
\text { mium- } \\
\text { Zahl } \\
\text { mg }\end{array}$ & $\begin{array}{c}\text { Titra- } \\
\text { tion }\end{array}$ & $\begin{array}{c}\text { Cad- } \\
\text { mium- } \\
\text { Zalil } \\
\text { mg } \\
\end{array}$ & $\begin{array}{l}\text { Titra- } \\
\text { tion }\end{array}$ & $\begin{array}{c}\text { Cad- } \\
\text { mium- } \\
\mathrm{Zahl} \\
\mathrm{mg}\end{array}$ & $\begin{array}{c}\text { Titra- } \\
\text { tion }\end{array}$ & $\begin{array}{c}\text { Cad- } \\
\text { mium- } \\
\text { Zahl } \\
\text { mg }\end{array}$ \\
\hline $\begin{array}{l}\text { Altdorf bei Nürn- } \\
\text { berg, Gut II } \\
(=\text { No.7 derTab.I) }\end{array}$ & 28,3 & 222,7 & 8,6 & 70,4 & 9,2 & 108,0 & 11,2 & 121,0 & 12,5 & 150,2 & 13,5 & 179,0 \\
\hline $\begin{array}{c}\text { Herzogenaurach } \\
\text { (Oberfranken) } \\
\text { (= Nr. } 5 \text { der Tab.I) }\end{array}$ & 22,2 & 224,2 & 8,4 & 82,0 & 10,5 & 116,0 & 11,4 & 129,0 & 13,0 & 167,4 & 14,1 & 183,4 \\
\hline $\begin{array}{l}\text { Radstädter Tauern } \\
\text { (Salzburg), Gut II } \\
\text { Kuh S. (= No. } 32 \\
\text { der Tab. I) }\end{array}$ & 32,3 & 230,6 & 8,6 & 90,0 & 10,4 & $\mathrm{I} 21,0$ & 12,0 & 134,0 & 13.5 & 161,0 & 13,9 & 185,9 \\
\hline
\end{tabular}

[Fortsetzung von S. 29.]

Die Titrationswerte ermöglichen auch einen Vergleich der Mengen von schwerlöslichen, flüchtigen Fettsäuren mit den nach dem Verfahren von Polenske ${ }^{2}$ ) erbaltenen. Erstere Werte bewegen sich nach Tabelle I zwischen 5,9 und 9,2, während die Polenske'schen Zahlen normaler Butterfette mit steigender Reichert-Mei Bl'scher Zahl zwischen 1,35 und 3 liegen, wobei zu berïcksichtigen ist, daßs bei unserem Verfahren nur die Häfte der für die Bestimmung der Polenske'schen Zahl erforderlichen Fettmenge angewendet wird, während die Daver der Destillation und die Menge des Destillates in unserem Falle annähernd doppelt so groß sind, wie bei dem Verfahren von Polenske. Wie sich aus dem Vergleich obiger Zahlen ergibt, werden durch Destillation im Wasserdampfstrome weit größere Mengen der schwerlöslichen Säuren verflüchtigt wie nach dem einfachen Destillationsverfahren.

Wir haben uns auch mit der Frage beschäftigt, welche flüchtigen Fettsäuren an der Bildung der unlöslichen, bezw. schwerlöslichen Cadmiumsalze, wie sie nach unserer vorstehend beschriebenen Methode erbalten werden, überwiegend

1) Palmin der Tabelle II.

2) Diese Zeitschrift 1904, 7, 273. 
beteiligt sind. Die Beantwortung dieser Frage ist mit Schwierigkeiten verbunden, denn es gibt zuxzeit kein Verfahren, um aus einem Gemisch mehrerer, einander nahe stehender Fettsäuren die einzelnen Bestandteile quantitativ zu isolieren. Der einzige Weg, der unter-Umständen zu einer jedoch keineswegs quantitativen Trennung und Bestimmung eingeschlagen werden könnte, ist das kombinierte Verfahren der fraktionierten Destillation und Fällung. Derartige Versuche erfordern aber einen sehr großen Aufwand an Material und Zeit, der nicht im Verhältnis zu dem zu erwartenden Erm gebnis stände. Wir haben uns daher begnügt, auf einem anderen indirekten Wege etwas über die Zusammensetzung des Cadmiumsalzgemisches zu erfahren. Dies konnte dadurch geschehen, daß man feststellte, welche von den in Betracht kommenden flüchtigen Fettsäuren als Alkalisalze unter den angegebenen Versuchsbedingungen durch Cadmiumsulfat fällbar sind, und durçh die Bestimmung des mittleren Molekulargewichts des an der Cadmiumfällung beteiligten Fettsäuregemisches aus Butter- und Cocosfett bezw. Mischungen derselben. Das mittlere Molekulargewicht kann auf einfache Weise aus dem Cadmiumgehalt der Cadmiumsalzfällung berechnet werden unter der Voraussetzung, daß bei der Fällung keine basischen, sondern ausschließlich normale Cadmiumsalze entstehen. Unsere dahin zielenden Versuche sind noch nicht abgeschlossen, doch hoffen wir bald darüber berichten zu können.

Um die Löslichkeitsverhältnisse der Cadmiumsalze der flüchtigen Fettsäuren, wie sie auf Zusatz von Cadmiumsulfat zu den Kaliumsalzen der betreffenden Säuren erhalten werden, kennen zu lernen, wurden von den von C. A. F. Kahlbaum-Berlin bezogenen reinen Fettsäuren bestimmte Mengen in Verhältnissen, wie sie in den Destillaten ans Butter und Butter-Cocosfett-Gemischen vorkommen können, abgewogen, in einigen Tropfen Alkohol gelöst, mit Kalilauge neutralisiert, mit destilliertem Wasser auf $50 \mathrm{ccm}$ verdännt und mit je $2 \mathrm{~cm}$ einer 20\% 0 -igen Cadmiumsulfatlösung gefallt. Der Niederschlag warde in einem Gooch-Tiegel abgesaugt, mit. $50 \mathrm{ccm}$ Wasser in kleinen Anteilen ausgewaschen und bei $100-110^{\circ}$ getrocknet und gewogen.

Für die Versuche verwendeten wir Butter-, Capron-, Capryl-, Capriaund Laurinsäure.

Aus der nachfolgenden Tabelle IV No. 1-4 sind die Löslichkeitsverhältnisse der Cadmiumsalze der genannten Säuren für sich und in Gemischen ersichtlich.

Tabelle IV.

Versuch No. 1: Konzentration der Säuren : 0,025 g : $50 \mathrm{ccm}$.



Versuch No. 2: Konzentration der Säuren: $0,05 \mathrm{~g}: 50 \mathrm{ccm}$.

Buttersäure

Capronsäure

Caprylsäure

Caprinsäure

Laurinsäure

N. 09 .

Keine Fallung
Keine Fällang
$0,0185 \mathrm{~g}$
$0,0630 \mathrm{~g}$
$0,0620 \mathrm{~g}$

$\begin{array}{cc}- & - \\ 0,0698 & g \\ 0,0664 & \mathrm{~g} \\ 0,0641 \mathrm{~g}\end{array}$



3 
Versuch No. 3: Konzentration der Säuren: $0,1: 50 \mathrm{ccm}$.

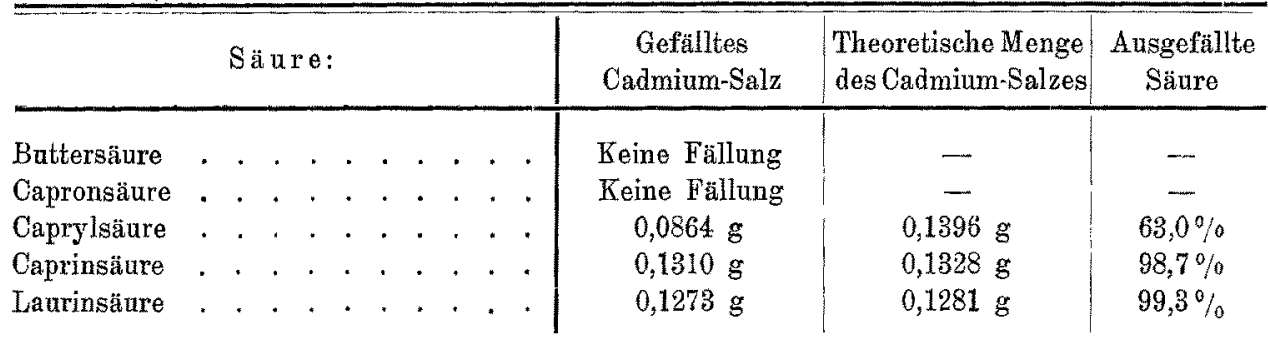

Versuch No. 4. Fettsäure-Gemische.

a) Konzentration der Säuren: Je 0,025 g: $50 \mathrm{ccm}$.

\begin{tabular}{|c|c|c|}
\hline \multirow[b]{2}{*}{ Säure Gemisch } & \multicolumn{2}{|c|}{ Menge der Cadmiumsalze } \\
\hline & Gefunden & $\begin{array}{c}\text { berechnet aus } \\
\text { Tabelle IV, } 1 \text { bezw. } 2\end{array}$ \\
\hline  & $\begin{array}{l}0,0062 \mathrm{~g} \\
0,0436 \\
0,0800 \\
0,0432 \\
0,0786 \\
0,0624\end{array}$ & $\begin{array}{l}0,0040 \mathrm{~g} \\
0,0317 \\
0,0621 \\
0,0317 \\
0,0621 \\
0,0581\end{array}$ \\
\hline
\end{tabular}

b) Konzentration der Säuren: Je 0,05 g: $50 \mathrm{ccm}$.

\begin{tabular}{l|l|l|l} 
Capron- + Caprylsäure . . . . . . . . . . . . & $0,0246 \mathrm{~g}$ & $0,0185 \mathrm{~g}$ \\
Capron- + Capryl- + Caprinsäure. . . . . . . & $0,1010 \%$ & $0,0815 \%$
\end{tabular}

Buttersäure und Capronsäure werden demnach unter den an. gegebenen Versuchsbedingungen nicht gefälit, ihre Cadmiumsalze sind in Wasser leicht löslich. Das Cadmiumsalz der Caprylsäure ist schwerer löslich und scheidet sich zum kleineren Teile aus, wenn die Säure in Konzentrationen von $0,025 \mathrm{~g}: 50 \mathrm{ccm}$ und von $0,05 \mathrm{~g}: 50 \mathrm{ccm}$ zur Anwendung gelangt. Dagegen werden Caprin- und Laurinsäure fast vollständig als Cadmiumsalze gefällt; so

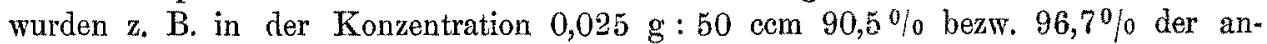
gewandten Säuren als Cadmiumsalze abgeschieden. Von der Caprylsäure gehen bei der gleichen Konzentration 11,5\%, bei der zweifachen $13,2 \%$, bei einer Konzentration von $0,1 \mathrm{~g}: 50 \mathrm{ccm}$ dagegen schon $63 \%$ der Säure als Cadmiumsalz in den Niederschlag. Anders liegen die Verhältnisse, wenn es sich, wie bei den von uns erhaltenen Destillationen aus Butter- und Cocosfett oder Gemischen derselben, um Mischungen der Fettaäuren handelt. In diesen Fällen werden die Mengen der ausfallenden Cadmiumsalze größer sein wie die Summe der Cadmiumfällungen der einzelnen Säuren für sich, entsprechend der Regel, daß die Löslichkeit eines Salzes bei Gegenwart eines zweiten mit gemeinschaftlichem Ion sinkt. Die Versuche No. $4 \mathrm{a}$ und b der Tabelle IV mit Mischungen mehrerer Fettsäuren in bestimmten Mengenverhältnissen lassen dieses Verhalten deutlich erkennen.

Aus den 4 Versuchsreihen der Tabelle IV geht ferner hervor, daß an der $\mathrm{Zu}$ sammensetzung der Cadmiumsalz-Fällungen, wie sie nach unserem Verfahren aus 
Butterfett und Butterfett-Cocosfett-Mischungen erbalten werden, buttersaures und capronsaures Cadmium nicht beteiligt sein können, da diese Salze wasserlöslich sind. Dazu kommt, daß die freien Säuren wegen ihrer relativ leichten Löslichkeit in Wasser schon zum größten Teil bei der Filtration der nach der Verseifung der Fette erhaltenen festen Säuren entfernt werden. Sie könnten nur soweit überhaupt in das Destillat gelangen, als geringe Mengen der beiden Säuren dem festen Säuregemisch anhaften bezw. von ihm mechanisch eingeschlossen werden.

Bestimmt werden dagegen an der Entstehung der Cadmiumsalafällung beteiligt sein Caprin- und Caprylsäure, erstere nahezu in ibrer Gesamtheit, letztere nur teilweise, abhängig von der Konzentration der Lösung und der Menge der übrigen flüchtigen Fettsäuren.

Weiter kommen noch in Betracht Laurin- und Myristinsäure, da sie unter Anwendung unseres Destillationsverfahrens mit Wasserdämpfen, wenn auch ziemlich schwer, flüchtig sind und unlösliche Cadmiumsalze bilden. In welchen Mengen die beiden Säuren in den Cadmiumsalzällungen vorhanden sind, darüber könnte nur die Bestimmung des mittleren Molekulargewichts der an Cadmium gebundenen Säurereste einige Anhaltspunkte ergeben. Wie schon erwähnt, sind unsere diesbezüglichen Versuche noeh nicht abgeschlossen.

Da die in Tabelle I angeführten Cadmium-Zahlen den Wert 90 nicht überstiegen, obwohl die Milchfettproben, von denen diese Zahlen bestimmt wurden, in der Reichert-Meißl'schen Zahl und Verseifungszahl unter sich weit auseinander liegende Werte ergaben und auch die das Milchfett liefernden Tiere nach Rasse, Fütterungsart und Laktation erhebliche Verschiedenheiten aufwiesen, so hofften wir anfangs, nach Sammlung eines noch größeren Analysenmaterials schlieBlich für die praktische Beurteilung den Satz aufstellen zn könıen: Butterfette mit einer CadmiumZahl unter $100 \mathrm{mg}$ sind nicht zu beanstanden, solche mit einer Cadmium-Zahl über $100 \mathrm{mg}$ sind mit Cocosfett vermischt.

Die neue und neueste Literatur und die Erfahrungen mit der ,neuen Butterzahl" lehren aber, daß man in der Aufstellung von Grenzwerten ungemein vorsichtig sein muß, und man wird der Ansicht Lührig's') zustimmen müssen: „daß es durchaus nicht genügt, gegebenenfalls an einer Reihe reiner Produkte, sowie an einer Reihe künstlicher Mischungen die Probe aufs Exempel zu machen".

\section{Fütterungsversuche.}

Da wir heute recht wohl wissen, daB der Tierkörper unter gewissen Bedingungen ein Butterfett mit solchen Eigenschaften erzeugen kann, daß es als gefälscht im Sinne unserer Beurteilungsnormen zu gelten haben würde, so schien es uns auch hier notwendig, vor Aufstellung von Grenzzahlen auf den Tierversuch zurückzugreifen. Es unterliegt heute keinem $Z$ weifel mehr, daß Fütterungsart und eine vorgeschrittene Laktationszeit von großem Einflub auf die Bildung und Zusammensetzung des Milchfettes sind. So entstehen unter dem Einflusse von Cocoskuchen- und Rüben-Fütterung Milchfette mit großen Differenzen im Sinne Juckenack-Pasternack's und abnorm hohen Polenske'schen Zahlen, während eine vorgeschrittene Laktationsperiode einen Rückgang im Gehalt des Milchfettes an den Glyceriden der leichtflüchtigen Säuren mit sich bringt. Auf Grund dioser Erfahrungen hielten wir es für erforderlich, Fütterungsversuche vorzunehmen mit proteinreichen (Malzkeime)2), cocosfetthaltigen (Cocoskuchen)

1) Diese Zeitschrift 1906, 11, 15.

2) Ein unter dem Einfluf der Malzkeimfütterang produziertes Milchfott findet sich in Tabelle I. nnter Nr. 1. 
und zuckerreichen Futtermitteln (Rübenköpfe), außerdem auch von altmilchenden Kühen Milchproben zu entnehmen und von den so unter verschiedenen Verhältnissen und Einflüssen gewonnenen Milchfetten die Cadmium-Zahlen zu bestimmen. Daß diese hierbei größeren Schwankungen unterworfen sein würden, schien nicht zweifelhaft. Über die Grenzen, innerhalb welcher sich diese Schwankungen bewegen, geben die nachfolgenden Versuche Auskunft.

A. Fütterung mit Cocoskuchen.

Durch die Versuche von Baumert und Falke'), Lührig'), Siegfeld ${ }^{3}$ ), Dons $\left.{ }^{4}\right)$ u. a. ist festgestellt, daß bei Verfütterung von Cocoskuchen die Menge der im Milchfett enthaltenen Glyceride der löslichen flüchtigen und der hochmolekularen ungesättigten Säuren vermindert wird; dadurch fallen Reichert-MeiBl'sche Zahl und Jodzahl. Die Gruppe der Glyceride der in Wasser schwerlöslichen bezw. unlöslichen, mehr oder minder flüchtigen Säuren von mittlerem Molekulargewicht erfährt dagegen eine bedeutende Vermehrung, wodurch ein Ansteigen der Verseifungszahl bedingt wird. Die Juckenack-Pasternack'schen Differenzen zeigen demzufolge hohe negative Werte, weit höher als sie ursprünglich von diesen Forschern ${ }^{5}$ ) als äußerste Grenzwerte für reine Butter angegeben wurden. Die Jucken ack-Pasternack'sche Differenz ist seinerzeit von $\mathrm{Lührig}$ auf Grund seiner Versuchsergebnisse als ungeeignet für die Beurteilung der Reinheit von Butterfett angesehen worden. Es hat sich aber gezeigt, und auch aus unseren nachfolgenden Versuchen geht dies hervor, daß auch die anderen neuen Methoden einschließlich der Polenske'schen ZahI jede für sich allein genommen, versagen, wenn sie an reinen Butterfetten mit hohen negativen Jucken ack-Pasternack'schen Differenzen erprobt werden. Für die Polenske'sche Zahl z. B. ergab sich nach Versuchen von Lührig und Dons, daß bei Butterfetten, die von reichlich mit Cocoskuchen gefütterten Tieren stammten, Reichert-Meifl'sche Zahlen und Polenske'sche Zahlen nicht mehr in dem angenommenen normalen Verbältnis standen, sondern daß den hohen Differenzen auch abnorm hohe Polenske'sche Zahlen entsprachen.

Unsere Versuche bestätigen dieses Ergebnis ebenfalls.

Die Ausführung der von uns in Altdorf bei Nürnberg veranlaßten Fütterungsversuche geschah in folgender Art:

Als Versuchstiere wurden 2 Kühe gewählt, die altmilchend, 26-30 Wochen trächtig waren und demnach Milchfette mit relativ niedrigen Rei chert-Mei $\mathrm{l}$ l'schen Zahlea erzeugten. Es wurden absichtlich altmilchende Kühe genommen, um auch zugleich hinsichtlich der sogenannten Butterkonstanten die von Lührig u. a. beschriebenen Versuche zu ergänzen, bei denen Milchfette mit höheren Reichert-Meißl'schen Zahlen analysiert wurden, die Versuchstiere also frischmilchend waren.

Die einzelnen Rationen an Cocoskuchen wurden, ähnlich wie bei den Lührig'schen Versuchen bis zu der erreichbaren Höchstgabe gesteigert und den Kühen bis zu 10 Pfund für den Tag und Kopf neben einem aus Heu und Häcksel bestehenden indifferenten Futter verabreicht. Eine weitere Vermehrung des Kraftfutters war unmöglich, da die Tiere nach der 8. Periode die Aufnahme noch größerer Mengen verweigerten.

1) Diese Zeitschrift $1898,1,665$,

2) Diese Zeitschrift 1906, 11, 15 .

3) Milchwirtschaftl. Zentralblatt 1906, 2, 289.

d) Diese Zeitschrift 1907, 14, 341.

5) Diese Zeitschrift 1904, 7, 198. 
Die Versuche, die unter Aufsicht des Herrn Landwirtschaftslebrers We idring er in Altdorf standen, begannen mit einer Vorperiode ohne Cocoskuchen. Als Grundfutter dienten Gras, Heu und Häcksel. Am 3. Tag jeder Versuchsperiode wurden Morgen-, Mittag- und Abendmilch gesammelt, nach Érlangen gesandt, im Laboratorium aufrahmen gelassen, der Rahm am anderen Morgen in einer Haushaltungs-Buttermaschine ausgebuttert und das Butterfett durch Ausschmelzen bei $50-60^{\circ}$ von Wasser, Casein und Salzen befreit.

Die Reichert.Meißs'sche Zahl wurde nach dem gewöhnlichen Verfahren und nach Polenske bestimmt. Die "noue Butterzahl" ermittelten wir genau nach den Angaben von Polenske. Die Verseifungszahlen sind die arithmetischen Mittel von je drei innerhalb sehr geringer Grenzen schwankenden Bestimmungen.

Die in den einzelnen Fütterungsperioden bei den eingelieferten Proben gefundenen Zahlen sind in Tabelle $\mathrm{V}$ wiedergegeben.

Aus dem darin zusammengefaßten Analysen-Material ergibt sich folgendes:

Der Übergang der charakteristischen Bestandteile des Cocosfetts in das Milchfett vollzieht sich bei Verfütterung mäßiger Mengen von Cocoskuchen nur langsam und wird analytisch deutlich wahrnehmbar erst nach länger dauernder, intensiver Fütterung. Nach 15-tägiger Verabreichung von Cocoskuchen, nachdem bereits 34,5 Pfund auf das Tier verfüttert waren (4. Periode), ist hinsichtlich der ReichertMeißl'schen Zahl und der Verseifungazahl ein bedeutenderer Unterschied in der Zu* sammensetzung des Milchfettes vor und nach der Cocoskuchen-Fütterung nicht zu erkennen. Nennenswerte negative Juckenack-Pasternack'sche Differenzen treten erst in der 5. Periode auf und steigen in der 8. Periode bis zu-12,9 an, wo aber schon Cocoskuchen-Rationen in einer Höhe gegeben wurden, wie sie der praktische und haushälterische Landwirt wohl nicht verfüttert. Die Reichert-Meißl'sche Zahl sinkt verhältnismäßig langsam, im ganzen um etwa 2,5 Einheiten, dagegen findet bei reichlicher Cocoskuchenfütterung ein sehr beträchtliches Ansteigen der Verseifungszahlen statt,

\section{Tabelle V.}

Fütterungsersuche mit Cocoskuchen (Mittlerer Fettgehalt 18,7\%) ausgeführt im Oktober 1907.

\begin{tabular}{|c|c|c|c|c|c|c|c|c|c|c|c|c|}
\hline \multirow{2}{*}{$\begin{array}{c}\text { Fütte- } \\
\text { rungs- } \\
\text { Perioden }\end{array}$} & \multirow{2}{*}{\multicolumn{2}{|c|}{$\begin{array}{l}\text { Menge der } \\
\text { Cocoskuchen- } \\
\text { Rationen }\end{array}$}} & \multirow{2}{*}{ 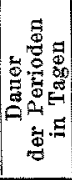 } & \multirow{2}{*}{ 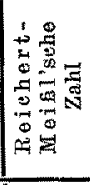 } & \multirow{2}{*}{ 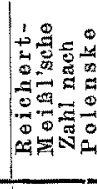 } & \multicolumn{2}{|c|}{$\begin{array}{c}\text { Polenske- } \\
\text { sche Zahl }\end{array}$} & \multirow{2}{*}{ 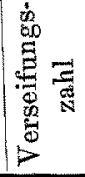 } & \multirow{2}{*}{  } & \multirow{2}{*}{  } & \multicolumn{2}{|c|}{$\begin{array}{l}\text { Cadminm- } \\
\text { Zahlen (mg) }\end{array}$} \\
\hline & & & & & & $\begin{array}{l}\text { ge. } \\
\text { funden }\end{array}$ & & & & & $\begin{array}{c}\text { des } \\
\text { reinen } \\
\text { Butter- } \\
\text { fottes } \\
\end{array}$ & $\begin{array}{l}\text { d. Butter- } \\
\text { fettes mit } \\
10 \% \\
\text { Cocosfett } \\
\end{array}$ \\
\hline periode ${ }^{2}$ ) & $\begin{array}{l}\text { Norm } \\
\text { Fütt }\end{array}$ & & 3 & , & 22,0 & 1,5 & 1,5 & 1,4 & $+0,9$ & 7,4 & 83 & 11 \\
\hline$\left.I^{3}\right)$ & $y_{5}$ & Pfd. & 6 & 23,5 & 28,3 & 1,5 & $1,5-1,7$ & 221,9 & $+1,6$ & 8,0 &  & 1 \\
\hline$\left.I I^{*}\right)$ & 0 & & 3 & 22,7 & 22,1 & 1,7 & 1,5 & 221,0 & $+1,7$ & 7,9 & 76,4 & 112,2 \\
\hline$\Pi$ & 2,5 & & 3 & 22,0 & 21,7 & 2,1 & $1,4-1,5$ & 222,8 & $-0,8$ & - & 83 & - \\
\hline II & 4 & & 3 & 19 & 19, & 2 & 1,35 & 228,8 & $-4,2$ & 8,8 & 87 & - \\
\hline $\mathrm{V}$ & 6 & & 3 & 21,9 & 22,0 & 2,8 & $1,4-1,5$ & 229,0 & $-7,1$ & - & 9 & - \\
\hline V & 7 & & 3 & 21,5 & 21,5 & 2,5 & $1,4-1,5$ & 230,2 & $-8,7$ & 9,2 & 10 & - \\
\hline & 8 & & 3 & 19,9 & 19,9 & 2,7 & 1,35 & 232,4 & $-12,5$ & 11,8 & 10 & - \\
\hline VIII & 10 & & 6 & 19,8 & 19,7 & 2,7 & 1,35 & 232,7 & $-12,9$ & 12,1 & 112 & - \\
\hline IX & $\begin{array}{r}\text { Normale } \\
\text { rung }\end{array}$ & Futit & - & 20,3 & 20,6 & 1,2 & - & 218,2 & $+2,1$ & 7,1 & 72 & - \\
\hline
\end{tabular}

1) Vergl. S. 29.

2) Versuch No. 4 der Tabelle I.

3) Versuch No. 8 der Tabelle I.

4) Versuch No. 3 der Tabelle I. 
Die Polenskes sche Zahl wird, unabhängig von der Reichert-Meiß1'sehen $\mathrm{Zahl}$, parallel mit der negativen Jucken ack-Pastern a ck'schen Differenz erhöht.

So mübte z. B. unter normalen Verbältnissen die nach der 7. Periode gefundene Polenske'sche Zahl von 2,7 einer Reichert-Meibl'schen Zahl von 29-30 entsprechen, tatsächlich wurde aber eine solche von 19,9 gefunden.

Unsere Befunde der Tabelle $\mathrm{V}$ decken sich mit den Ergebnissen der erwähnten Arbeiten von Lührig, Siegfeld und Dons. Ersterer studierte bei seinen Fütterungsversuchen den Einflub möglichst großex Cocoskuchengaben und fand dieselben großen Abweichungen von den normalen Werten, wie auch wir sie in den Fütterungsperioden VI-VIII ermittelten. Dagegen verfütterte Siegfeld nur bis zu 4 Pfund Cocoskuchen in lange dauernden Perioden. Fr fand für die Reichert-Mei Bl'schen Zahlen und die $\mathbf{P}$ olensk e'schen Zahlen keine größeren Abweichungen von der Norm, wohl aber sehr hohe Verseifungszahlen, von 226-236 ansteigend. S i e $\mathrm{f}$ el d schreibt diese Erhöhung der Verseifungszahlen dem Einfluß der Cocoskuchen-Fütterung zu. Seine Versuche sind aber in dieser Hinsicht nicht beweisend, denn zugleich mit den Cocoskuchen erhielten die Versuchstiere bis zu 50 Pfund Rübenschnitzel, deren Verfütterung, wie durch neuere Versuche festgestellt ist, zu Milchfetten mit hohen Verseifungszahlen führt.

Was die Cadmium-Zahl betriff, so fanden wir auch bei ihr parallel mit der Erhöhung der Cocoskuchengaben ein Ansteigen der Werte, doch macht sich dieser Einfluß bei mäßiger Verfütterung von Cocoskuchen nicht in gleichem Maße bemerkbar wie bei der Polenske'schen Zahl. So liefern die bei Cocoskuchenfütterung erhaltenen Butterproben bis einschließlich der Periode $V$ noch normale, d. h. unter 100 liegende Cadmium-Zahlen, wäbrend, nach den übrigen Konstanten beurteilt, das Fett der Periode V schon anormal erscheint. Erst bei sebr reichlicher Cocoskuchenfütterung, wie sie praktisch kanm vorkommt, erreicht die Cadmium-Zahl Werte, die einen absichtlichen Zusatz von etwa $10 \%$ Cocosfett zu dem betreffenden Butterfette vortäuschen könnten. Berücksichtigt man aber, daß diese hohen Zahlen Hand in Hand mit sehr hohen negativen Juckenack-Pasternack'schen Differenzen gehen, so erscheint, wie später näher ausgeführt werden soll, auch unter diesen Umständen eine richtige Beurteilung derartiger, hohe Cadmium-Zahlen liefernder Butterfette möglich.

\section{B. Fütterung mit Rüben.}

Die vor mehr als Jahresfrist erschienen Arbeiten von M. Siegfeld ${ }^{1}$ ) und dem einen von uns (A mberger $\left.{ }^{2}\right)$ ) über den Einfluß derRübenfütterung a uf die Zusammensetzung des Butterfettes haben ergeben, daß durch dieses Futtermittel das Mischungsverbältnis der das Milchfett zusammensetzenden Glyceride recht erbeblich verändert wird. Analytisch gibt sich diese Veränderung durch höhere Reichert-Meibl'sche Zahlen und Verseifungszahlen, mehr oder minder hohe negative Juckenack-Pasternack'sche Differenzen, abnorm erhöhte Polenske'sche Zahlen und durch ungewöhnlich niedrige Jodzablen zu erkennen. Daraus läbt sich auf eine Vermehrung der Glyceride der löslichen und unlöslichen flüchtigen wie auch der schwerflüchtigen Säuren (Laurin- und Myristinsäure) schließen. Daß die Glyceride der letzteren Säuren eine beträchtliche Zunahme unter dem Einfluß der Rübenfütterung erfahren, ergibt sich aus den hohen Verseifungszahlen. Hand in Hand damit geht

1) Diese Zeitschrift $1907,13,513$.

2) Diege Zeitschrift 1907, 13,614. 
die Verminderung des Glycerides der Ölsäure, analytisch nachweisbar durch die $\mathrm{Ab}$ nahme der Jodzahl.

Was schlieblich die bei Rübenfütterung beobachteten hohen Polenske'schen Zahlen betrifft, so weisen sie darauf hin, daß gerade die Glyceride der unlöslichen flüchtigen Säuren gegenüber den löslichen unverhältnismäßig unter der Wirkung des erwähnten Futtermittels zunehmen.

Im Gegensatz zu den bei Cocoskuchenfütterung sich ergebenden Butterfetten liegen für die praktische Beurteilung eines Butterfettes hier die Verhältnisse etwas günstiger, weil mit dem Steigen der Verseifungszahl auch ein langsames Ansteigen der Reichert-Meißl'schen Zahl verbunden ist und erst dann hohe negative J u cken ack-Pastern a ck'sche Differenzen auftreten, wenn dieReichert-Meibl'sche Zahl schon eine Höhe erreicht hat, bei der, wie dies auch Siegfeld betont, eine Beanstandung nicht mehr erfolgen sollte.

Freilich ist es nicht ausgeschlossen, dafo gerade derartige Butterfette mit ReichertMeißl'schen Zahlen über $30 \mathrm{za}$ Fälschnngen verlocken könnten. So würde z. B. ein Zusatz von 10-20\% Cocosfett die Reichert-Meißl'sehe Zahl nur um etwa 1,5-3,5 Einheiten herabdrücken. Bei orientierender Untersuchung, die sich vielleicht nur auf die Bestimmung der Reichert-Meißl'schen Zahl beschränkt, was ja anch heutzutage nicht allzuselten vorkommt, werden derartige Fälsehungen dem Nachweis entgehen. In der Praxis werden allerdings derartige Fälschungen mit Rüeksicht auf die Seltenheit von Butter mit ReichertMeißl'schen Zahlen üb er 30 nicht hänfig vorkommen.

Unsere in den folgenden Tabellen VI, VII und VIII (S. 40-41) angeführten Untersuchungsergebnisse beziehen sich auf Butterfette, die unter dem Einflusse mehr oder minder intensiver Rübenfütterung erzeugt wurden. Die Tabelle VI enthält u. a. das analytische Material einer Anzahl von Butterfetten aus norddeutsehen Molkereien, die wir durch freundliche Vermittelung der Herren Dr. W. Leuze-Dessau, Dr. Zitelmann-Berlin und Dr. W. Fuchs-Berlin erhielten. Im Anschluss an diese Untersuchung haben wir in Altdorf bei Nürnberg Fütterungsversuche unter Aufsicht des Herrn Landwirtschaftslehrers Weidringer an frisch-und altmilchenden $\mathrm{Kühen}$ anstellen lassen. Die Tabelle VII enthält die Versuchsergebnisse von Butterfetten der mit Rüben gefütterten frisehmilchenden $\mathrm{K} u ̈ h$ e, Tabelle VIII bezieht sich auf die Untersuchung der von altmilchenden, mit Rüben gefütterten Tieren stammenden Butterfette.

Die zur Untersuchung dienenden Milchproben wurden im Laboratorium entrahmt und verbuttert.

Von den einzelnen Butterproben wurde ermittelt:

1. Die Reichert-Meißl'sche Zahl, sowohl nach der gewöhnlichen Methode, als auch nach der Vorschrift von Polenske. - 2. Die Polenske'sche Zahl. - 3. Die Verseifungszahl und zwar von jeder Probe drei Bestimmungen, deren Mittelwert in den Tabellen angeführt ist. - 4. Die Juckenack-Pasternack'sche Differenz. - 5. Die Jodzahl. - 6. Die Titrationswerte der überdestillierten flüchtigen Säuren und die Cadmium-Zahlen sowohl der reinen Butterfette, als auch, in den meisten Fällen, der mit 10\% Cocosfett versetzten Butterfette.

In der erwähnten Abhandlung des einen von uns sind für den größeren Teil der aus Molkereien stammenden Butterproben, wie auch für die bei unseren $R$ übenfütterungsversuchen an frischmilchenden Kühen gewonnenen Butterfette Reichert-Meißl'sche Zahlen, Verseifungszahlen, Polenske'sche und Jodzablen schon 
Tabelle VI. Molkereibutter

\begin{tabular}{|c|c|c|c|c|c|}
\hline No. & Herkunft & Art der Fütterung & $\begin{array}{l}\text { Zuit der } \\
\text { Entnahme }\end{array}$ & $\begin{array}{c}\text { Reichert- } \\
\text { Meifl'sehe } \\
\text { Zahl }\end{array}$ & $\begin{array}{l}\text { Reichert- } \\
\text { Meisl'sehe } \\
\text { Zabl nach } \\
\text { Polenske } \\
\text { bestimmt }\end{array}$ \\
\hline 1 & Burglengenfeld (Oberpfalz) & $\begin{array}{c}\text { Trockenfutter, } \\
\text { Rüben Cocoskuchen }\end{array}$ & April 1907 & 22,2 & 22,6 \\
\hline 2\{ & $\begin{array}{c}\text { Königsberg } \\
\text { (Prov. Brandenburg) }\end{array}$ & $\begin{array}{c}\text { Wiesen- und Kleeheu, } \\
\text { Schlempe, Rüben }\end{array}$ & Oktober 1906 & 27,1 & 27,4 \\
\hline 3 & Kochstedt (Anhalt) & $\begin{array}{c}\text { Rüben, Rübenblätter, } \\
\text { Cocoskuchen }\end{array}$ & Novemb. 1906 & 27,1 & 27,4 \\
\hline 4 & Segrehna (Anhalt) & Heu, Rüben & & 28,2 & 28,3 \\
\hline 5 & Quellendorf (Anbalt) & Zuckerrüben-Abfälle & & 29,3 & 29,5 \\
\hline 6 & Molkerei Dessan & Zuckerrüben-Abfalle & , & 30,4 & 29,9 \\
\hline 7 & Lausigk (Anhalt) & Zuckerrüben-Abfälle & & 30,1 & 29,9 \\
\hline 8\{ & $\begin{array}{l}\text { Gut I, AItdorf } \\
\text { (Mittelfranken) }\end{array}$ & Rüben & Novemb. 1907 & 31,1 & 31,2 \\
\hline
\end{tabular}

Tabelle VII. Rübeufüterungsversuche bei

\begin{tabular}{|c|c|c|c|c|c|c|}
\hline \multirow{2}{*}{ No. } & \multirow{2}{*}{$\begin{array}{c}\text { Gröse der Rationen } \\
\text { für den Tag und Kopp }\end{array}$} & \multirow{2}{*}{$\begin{array}{l}\text { Dauer der } \\
\text { Fütterungs- } \\
\text { Perioden } \\
\text { in Tagen }\end{array}$} & \multirow{2}{*}{$\begin{array}{c}\text { Reichert- } \\
\text { Meikl'sche } \\
\text { Zabl }\end{array}$} & \multirow{2}{*}{$\begin{array}{l}\text { Reichert- } \\
\text { Meibl'sche } \\
\text { Zahl nach } \\
\text { Poleaske }\end{array}$} & \multicolumn{2}{|c|}{ Polensko-Zahl } \\
\hline & & & & & gefunden & normal \\
\hline $\left.1^{3}\right)$ & $\left\{\begin{array}{c}75 \text { Pfd. Runkelrüben } 1 \text { ) } \\
16,5 \text { Pfd. gekouhte } \\
\left.\text { Dorschen }{ }^{2}\right) \\
8 \text { Pfd. Malzkeime }\end{array}\right.$ & 3 & 31,0 & 31,2 & 3,6 & - \\
\hline 2 & (15 Pfd. Dorschen & 2 & 25,8 & 26,0 & 1,9 & 1,9 \\
\hline 3 & $=60 \%$ Rüben & 3 & 27,9 & 28,0 & 2,9 & $2,2-2,3$ \\
\hline 4 & \begin{tabular}{l|l} 
& 75
\end{tabular} & 3 & 29,4 & 29,5 & 3,4 & $2,6-2,7$ \\
\hline 5 & $\overrightarrow{F_{0}}, 90$ & 6 & 30,0 & 29,9 & 3,6 & $2,5-3,0$ \\
\hline 6 & \begin{tabular}{l|ll}
\multirow{3}{*}{} & 45
\end{tabular} & 3 & 28,6 & 28,9 & 2,8 & 2,5 \\
\hline 7 & 15, & 3 & 27,0 & 27,2 & 2,3 & 1,9 \\
\hline 8 & $15 *$ Dorschen & 3 & 26,4 & 26,5 & 1,8 & 1,9 \\
\hline
\end{tabular}

Tabelle VIII. Rübenfütterungs.Versuche

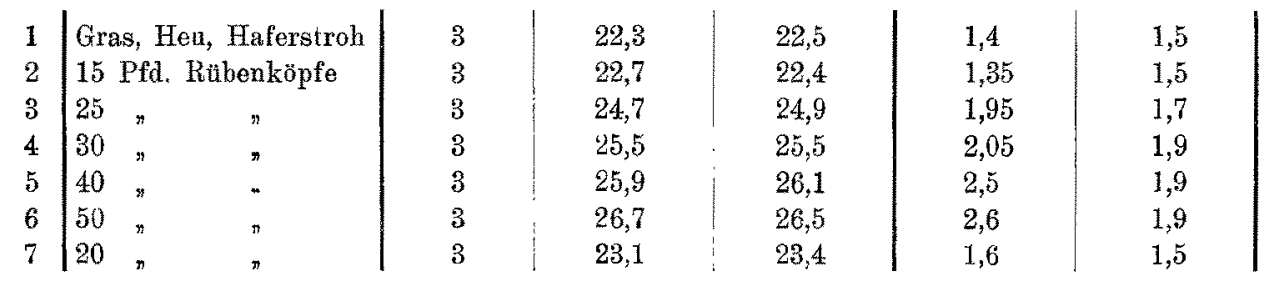

1) Die Rüben waren Eckendorfer Futterrunkeln (87,0\% Wasser, 5,7\% Zucker, 1,34\% Stickstoff-Substanz).

${ }^{2}$ ) Dorschen $==$ Wruken (zuckerarme Kohlrübenart).

s) Periode $I=$ vor Beginn der Versuche übliche Fütterungsart. 
bei Räbenfätterung.

\begin{tabular}{|c|c|c|c|c|c|c|c|c|}
\hline \multicolumn{2}{|c|}{ Polenske'sche Zahl } & \multirow{2}{*}{$\begin{array}{l}\text { Ver- } \\
\text { seifungs- } \\
\text { zahl }\end{array}$} & \multirow{2}{*}{$\begin{array}{c}\text { Jucke- } \\
\text { nack-Pa- } \\
\text { sternack' } \\
\text { sche } \\
\text { Differenz }\end{array}$} & \multirow[b]{2}{*}{ Jodzahl } & \multicolumn{2}{|c|}{ Titrationswert } & \multicolumn{2}{|c|}{$\mathrm{Cadmium \cdot Zah1}$} \\
\hline gefundeu & normal & & & & Butterfett & $\begin{array}{c}\text { Butterfett } \\
\text { mit } 10 \% \text { Co } \\
\text { cosfott }\end{array}$ & Butterfett & $\begin{array}{l}\text { Butiterfett } \\
\text { mit } 10 \% \text { Co- } \\
\text { cosfett }\end{array}$ \\
\hline 2,1 & 1,5 & 229,6 & $-7,4$ & - & 7,3 & 8,6 & 90,0 & 124,0 \\
\hline 2,3 & 1,9 & 238,2 & $-6,1$ & 26,2 & 9,4 & 10,4 & 88,0 & 117,0 \\
\hline 2,8 & 1,9 & 236,5 & $-9,4$ & 22,5 & 9,2 & 11,4 & 112,0 & 141,0 \\
\hline 2,7 & 2,3 & 234,3 & $-6,1$ & - & - & - & 108,0 & - \\
\hline 3,4 & 2,5 & 237,3 & $-8,0$ & 23,0 & 9,8 & 11,4 & 122,0 & 154,0 \\
\hline 3,5 & $2,5-3$ & 236,5 & $-6,2$ & 23,4 & 9.7 & 11,7 & 117,0 & 146,0 \\
\hline 3,7 & $2,5-3$ & 235,2 & $-5,1$ & $\varepsilon 3,8$ & 9,2 & 10,3 & 111,0 & 144,0 \\
\hline 3,8 & - & $24,3,1$ & $-12,0$ & - & - & - & 129,6 & - \\
\hline
\end{tabular}

frischmilchenden Kühen. (Februar 1907.)

\begin{tabular}{|c|c|c|c|c|c|c|}
\hline \multirow{2}{*}{$\begin{array}{l}\text { Verseifungs } \\
\text { zahl }\end{array}$} & \multirow{2}{*}{$\begin{array}{l}\text { Juckenack- } \\
\text { Pasternack" } \\
\text { sche Differenz }\end{array}$} & \multirow[b]{2}{*}{ Jodzahl } & \multicolumn{2}{|c|}{ Titrationswert } & \multicolumn{2}{|c|}{ Cadminm-Zahl } \\
\hline & & & Butterfett & $\begin{array}{l}\text { Butterfett mit } \\
10 \% \text { Cocosfett }\end{array}$ & Butterfett & $\begin{array}{l}\text { Butterfett mit } \\
10 \% \text { Cocosfott }\end{array}$ \\
\hline 238,9 & $-7,9$ & 21,8 & 9,8 & - & 130,0 & - \\
\hline 227,8 & $-2,0$ & 30,3 & 8,4 & - & 96,0 & - \\
\hline 233,2 & $-5,3$ & 27,2 & 9,4 & - & 106,9 & - \\
\hline 237,0 & $-7,6$ & 23,3 & 9,6 & -- & 112,0 & - \\
\hline 238,7 & $-8,7$ & 21.8 & 9,8 & - & 117,0 & - \\
\hline 236,3 & $-7,7$ & 22,8 & 9,7 & - & 112,0 & - \\
\hline 232,3 & $-5,3$ & 25,2 & 9,1 & - & 92,0 & - \\
\hline 229,6 & $-3,2$ & 27,6 & 9,2 & - & 86,0 & - \\
\hline
\end{tabular}

bei altmilchenden Kühen. (Oktober 1907.)

\begin{tabular}{|l|l|l|l|r|r|r|}
220,0 & $+2,3$ & 42,9 & 7,4 & 9,1 & $\mathbf{7 6 , 4}$ & $\mathbf{1 1 2 , 2}$ \\
220,5 & $+2,2$ & 43,1 & 7,3 & 9,0 & $\mathbf{7 1 , 0}$ & $\mathbf{1 0 4 , 3}$ \\
223,6 & $+1,1$ & - & 8,4 & 9,8 & $\mathbf{7 7 , 0}$ & $\mathbf{1 1 0 , 0}$ \\
227,6 & $-2,1$ & 39,3 & 8,0 & 11,5 & $\mathbf{8 1 , 0}$ & $\mathbf{1 1 6 , 0}$ \\
228,6 & $-2,7$ & 36,9 & 9,2 & 10,5 & $\mathbf{8 6 , 0}$ & $\mathbf{1 2 4 , 0}$ \\
229,1 & $-2,4$ & 35,3 & 8,9 & $\mathbf{1 0 , 3}$ & $\mathbf{8 8 , 7}$ & $\mathbf{1 1 8 , 3}$ \\
221,8 & $+\mathbf{1 , 3}$ & 41,0 & $\mathbf{7 , 9}$ & $\mathbf{9 , 3}$ & $\mathbf{7 9 , 8}$ & $\mathbf{1 1 4 , 6}$
\end{tabular}


[Fortsetzung von S. 39].

veröffentlicht. Bezüglich der Einzelheiten der Versuche sei daher auf die erwähnte Arbeit verwiesen. Neu hinzugefügt sind in den folgenden Tabellen VI und VII die Juckenack-Pasternack'schen Differenzen, die Titrationswerte und die Cadmium-Zahlen. Wie aus beiden Tabellen ersichtlich ist, steigen mit den ReichertMeißl'schen Zahlen und Verseifungszahlen, Hand in Hand mit hohen negativen Juckenack-Pasternack'schen Differenzen, nicht nur die Polenske'schen sondern auch die Cadmium-Zahlen, diese bis zur Höhe von $130 \mathrm{mg}$ (Tabelle VII).

Leider war es uns nicht möglich, weder bei den Fütterungsversuchen noch aus Molkereien, Butterproben mit so hohen Reichert-Meißl'schen Zahlen, Verseifungszahlen und Polenske'schen Zahlen zu erhalten, wie sie Siegfeld veröffentlicht bat. Wir können daher auch nicht sagen, welche Werte die Cadmium-Zahlen bei Butterfetten mit Reichert-MeiRl'schen Zahlen über 35, und Polenske'schen Zahlen über 5 erreichen; daß sie unseren Höchstwert 130 übersteigen werden, ist wohl mit Sicherheit anzunehmen.

Von besonderem Interesse sind die Ergebnisse einer Untersuchung von Lührig ${ }^{1}$ ), der kürzlich bei der Prüfung von 18 unter dem Einfluß der Rübenfütterung erzeugten Butterfetten ebenfalls niedrigere Reichert-MeiBl'sche Zahlen und Verseifungszahlen (höchste Reichert-M ei B l'sche Zahl 31,5, höchste Verseifungszah1 238,5) als Siegfeld und überraschenderweise nur wenig erhöhte Polenskesche Zahlen beobachtete.

Das in Tabelle VIII angeführte analytische Material, Rübenfütterungsversuche an altmilchenden Tieren betreffend, verdient auch insofern einiges Interesse, als das Versuchsergebnis eine Frage beantwortet, die Siegfeld in seiner mehr erwähnten Arbeit ${ }^{2}$ ) aufwirft, nämlich: „Wie werden sich nun die Verhältnisse gestalten, wenn Kühe, deren Milchfett eine niedrige Reichert-Meißl'sche Zahl besitzt, z. B. altmilchende, mit Rüben gefüttert werden ?"

Obwohl bei unseren Fütterungsversuchen an altmilchenden Kühen die ReichertM e ißl'sche Zahl innerhalb weniger Tage von 22 auf 26, also um 4 Einheiten steigt, ist der Zuwachs an Glyceriden der flüchtigen unlöslichen Säuren, wie ihn die Polenske'sche Zahl zum Ausdruck bringt, im Verbältnis noch bedeutender, sodaß sich also auch in diesen Fällen, bei Butterfetten mit relativ niedriger ReichertMeißl'scher Zahl die Polenske'sche Zahl nicht mehr in den von Polenske angegebenen Normal- bezw. Grenzwerten bewegt (vergl. Tabelle VIII, Perioden 4 und 5 ).

Die Übereinstimmung der Reichert-MeiBl'schen Zahl mit der Verseifungszahl wie sie in der Juekenack-Pasternack'schen Differenz (Tabelle VIII) zum Ausdruck kommt, ist in diesem Falle nur eine zufällige, denn sie blieb auch nach starker Rübenfütterung nur deswegen noch innerhalb der Grenzen normaler Differenzen, weil die betreffenden Butterfette vor Beginn der Rübenfütterung verhältnismäßig hohe positive Differenzen zeigten.

Auch an der Cadmium.Zahl ist, wie schon erwähnt, der Einfluf der einseitigen Vermehrung der Glyceride der flüchtigen unlöslichen Säuren, bedingt durch Rübenfütterung, unverkennbar. Aber im Gegensatz zur Polenske'schen Zahl und anderen Konstanten erscheinen noch alle Butterfette, deren Reichert-MeiBl'sche Zabl

1) Pharmazeut. Zentralhalle 1907, 48, 1049 u. 1067.

2) Diese Zeitschrift 1907, 13, 514. 
nicht über 27 und deren Verseifungszahl nicht über 232-233 liegen, normal, wenn wir vorläufig als Grenzwert für normale Butterfette die Cadmium-Zahl 100 aufstellen ${ }^{1}$ ).

Charakteristische Belege hierfür sind die Butterfette No. 1 und 2 der Tabelle VI und No. 4, 5 und 6 der Tabelle VIII,

\section{EinfIuß der Laktationszeit.}

Durch eine Anzahl von Untersuchungen ist festgestellt, daß mit dem Voranschreiten der Laktationszeit die Menge der Glyceride der flüchtigen Säuren im Milchfett stetig abnimmt, also die Reichert-MeiBl'sche Zahl sinkt.

So fand $\mathrm{Nilson}^{2}$ ), daß die Reichert-Meifl'sche Zahl von 33,4 im ersten Monat der Laktation bis auf 25,42 im 14. Monat herabging.

Vieth ${ }^{3}$ ) gibt an, daß bei Butterproben aus zwei großen Molkereien infolge voranschreitender Labtation die Reichert-Meißl'schen Zahlen der Butterfette in dem einen Molkereibetrieb zwischen 30 und 22,8, in dem anderen zwischen 31,3 und 22,8 schwankten.

Ferner wiesen Spallanzani und $\mathrm{Pirri}^{4}$ ) sowie eine Anzahl anderer Forscher, in Übereinstimmung mit $\mathrm{Nilson}$ und Vieth auf die jetzt allgemein anerkannte Tatsache hin, daß die Reichert-Mei ol'sche Zahl im ersten Stadium der Laktation relativ hoch ist, gegen Ende derselben aber immer niedriger wird.

Auch nach dieser Richtung wurde von uns die Cadmium-Zahl geprüft und von einer Kuh, ebenfalls aus dem Stalle des Gutes in Altdorf, wo die schon beschriebenen Fütterungsversuche ausgeführt wurden, Milchproben in verschiedenen $\mathrm{Ab}$. schnitten der Laktationsperiode entnommen.

Die betreffende Kuh batte Ende November 1906 gekalbt, war gegen Ende Dezember 1906 belegt worden, hatte Ende Oktober 1907 wieder gekalbt und bis eine Woche vor dem Kalben Milch geliefert, stand also nicht oder nur ganz kurze Zeit trocken. Ails Futter erhielt sie zu den Zeiten der Probenentnahme bezw. immer schon 8 Tage vorher, Heu, Häcksel und geringe Mengen Treber, um eine Beeinflussung des Milchfettes durch die Fütterung möglichst auszuschalten. Da es nicht darauf ankam, den Einflus der Laktation anf die Zusammensetzung des Milchfettes in allen Einzelheiten festzustellen, schien es genügend, am Anfang, in der Mitte und gegen Ende der Laktationsperiode Milchproben zu entnehmen and die Cadmium-Zahlen der aus diesen Proben gewonnenen Milchfette zu bestimmen.

Die Versuchsergebnisse sind in der folgenden Tabelle IX angeführt. Die Untersuchung des Milchfettes einer zweiten altmilchenden Kuh (Tabelle IX, No. 5) aus der Umgebung von Erlangen lieferte ein mit den Proben aus Altdorf übereinstimmendes Ergebnis. Die Versuche haben im allgemeinen das eingangs Gesagte bestätigt. Sie ergaben ein Zurückgehen der Glyceride der flüchtigen löslichen und unlöslichen Säuren mit fortsehreitender Laktation.

1) Das Butterfett, für welches wir die niedrigste Cadmium-Zahl $(69,8)$ fanden, ergab, mit $10 \%$ Cocosfett vermischt, die Cadmium-Zahl 102. Diese Minimalwerte kommen aber praktisch nicht in Betracht, da es sich hierbei um das Milchfett einer einzelnen Kah im letzten Stadium der Laktatiou handelte. (Tabelle IX, No. 4.)

2) Zeitschr. analyt. Chem. 1889, 28, 175.

3) Milch.-Ztg. 1899, 28, 785.

4) Milch-Zitg. 1889, 18, 461 и. 483. 
Ungefähr gleichmäßig mit der Reichert-Meißl'schen Zahl sinkt die VerseifungsZahl, sodaß in ihrem Verhältnis zueinander keine anormalen Differenzen auftreten.

Die Cadmium-Zahl der Milchfette von beiden Tieren geht mit der Depression der Verseifungszabl etwas zurïck, hält sich aber noch in der 30. Woche der Laktation und in der 26.-27. Woche der Trächtigkeit des betreffenden Tieres nahe um 80 und sinkt erst im letzten Stadium der Laktation, als die Kuh 36-38 Wochen trächtig war, auf 69,8 herab. Die Tragezeit des Tieres betrug 40-41 Wochen. Selbstverständlich kommt derartige Milch, die nur in geringer Menge produziert wird, deren Geschmack zu wünschen übrig läßt und die sich auch nur schlecht oder gar nicht verbuttern läßt, praktisch nicht in Betracht.

So konnte die Milchfettprobe No. 4 der Tabelle IX nicht mehr durch Ausbuttern, sondern nur durch Extraktion mit Äther erhalten werden.

Die Änderungen in der Cad mi um-Zahl, wie sie die vorschreitende Laktation mit sich bringt, werden also der praktischen Beurteilung der Butterfette kaum Schwierigkeiten bieten.

Tabelle IX. Einfluk der Laktationszeit.

\begin{tabular}{|c|c|c|c|c|c|c|c|c|c|c|c|c|c|}
\hline \multirow[b]{2}{*}{ No. } & \multirow[b]{2}{*}{$\begin{array}{l}\text { Bezeichnang der } \\
\text { Kuh and der Zeit } \\
\text { der Probenentnahme }\end{array}$} & \multicolumn{2}{|c|}{$\begin{array}{l}\text { Latatations- } \\
\text { periode }\end{array}$} & \multirow[b]{2}{*}{  } & \multirow[b]{2}{*}{ 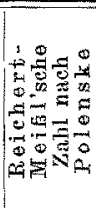 } & \multicolumn{2}{|c|}{$\begin{array}{c}\text { Polenske'- } \\
\text { sche Zahl }\end{array}$} & \multirow[b]{2}{*}{$\begin{array}{l}\text { Yersei- } \\
\text { fungs- } \\
\text { zahI }\end{array}$} & \multirow[b]{2}{*}{ 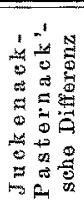 } & \multicolumn{2}{|c|}{$\begin{array}{l}\text { Titrations- } \\
\text { wert }\end{array}$} & \multicolumn{2}{|c|}{$\begin{array}{l}\text { Cadmium - } \\
\text { Zahl }\end{array}$} \\
\hline & & 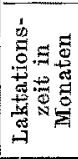 &  & & & 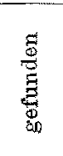 & 节 & & & 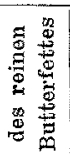 & 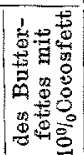 & $\begin{array}{c}\text { Butter- } \\
\text { feti }\end{array}$ & $\begin{array}{l}\text { Butter- } \\
\text { fott mit } \\
10 \% \\
\text { Oocos- } \\
\text { fett }\end{array}$ \\
\hline 1 & 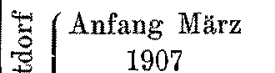 & 4 & 3 & 26,2 & 26,3 & 2,3 & 1,9 & 224,5 & $+1,7$ & 7,9 & 11,0 & 88,0 & 122,6 \\
\hline 2 & $\begin{array}{l}\text { Mitte Juli } \\
\quad 1907\end{array}$ & $7^{1 / 2}$ & $6^{1 / 2}$ & 24,2 & 24,6 & 1,9 & 1,7 & 224,9 & $-0,7$ & 8,5 & - & 81,2 & - \\
\hline 3 & \begin{tabular}{c|c} 
& Anfang Okto- \\
ber 1907 &
\end{tabular} & $9^{1 / 2}$ & $8^{1 / 2}$ & 23,3 & 23,5 & 1,7 & $1,5-1,7$ & 222,7 & $+0,6$ & 8,6 & 9,2 & 70,4 & 108,0 \\
\hline 4 & \begin{tabular}{c|c}
1907 & Mitte Oktober \\
& 1907
\end{tabular} & 10 & 9 & 23.1 & 23,0 & 1,6 & 1,6 & 220,6 & $+2,5$ & 7,9 & - & 69,8 & 102,0 \\
\hline$\tilde{5}$ & $\left\{\begin{array}{c}\text { Altmilchende Kuh } \\
\text { aus Tennenlohe } \\
\text { bei Erlangen }\end{array}\right\}$ & - & $7-8$ & 22,2 & 22,4 & 1,6 & 1,5 & 224,2 & $-2,1$ & 8,4 & 10,5 & 82.0 & 116,0 \\
\hline
\end{tabular}

\section{Die Beurteilung der Butterfette auf Grund der Cadmium-Zahl.}

In vorstehender Untersuchung sind zunächst von einer Reihe normaler Butterfette, geordnet nach ihren Reichert-MeiBl'schen Zahlen, von Butter-Cocosfettmischungen (Tabelle I und III) und von reinem Cocosfett (Tabelle II) die Cadmium-Zahlen bestimmt worden. Sie wurden ferner ermittelt von einer Anzahl anormaler Butterfette, wie sie unter dem Einfluß der Fütterung mit Cocoskuchen (Tabelle V) und Rüben (Tabelle VII und VIII) und im vorgeschrittenen Stadium der Laktation (Tabelle IX) erhalten werden. Auch haben wir reine Butterproben des Handels untersucht, die im Verhältnis zu ihren Reichert-Meibl'schen Zahlen ungewöhnlich hohe Verseifungszahlen aufwiesen (Tabelle VI). Wir bekamen derartige Proben aus den Gegenden, wo reichlich Cocoskuchen oder Rüben oder Mischungen beider verfüttert werden. Das so zusammengetragene analytische Material liefert zunächst einen 
weiteren Beweis für die großen Schwankungen in der Zusammensetzung der Butterfette, bedingt durch die großen Schwankungen in den relativen Mengenverhältnissen der im Milchfett enthaltenen Glyceride der löslichen und unlöslichen, leicht- und schwerflüchtigen Fettsäuren (bis zur Myristinsäure einschließlich) und der hochmolekularen ungesättigten Fettsäuren.

Aus den auf unsere Veranlassung angestellten Fütterungsversuchen erkennt man aufs neue, daß es möglich ist, durch bestimmte Futtermittel auf den tierischen Organismus so einzuwirken, daß im Milchfett bestimmte Gruppen von Glyceriden vermehrt oder vermindert werden. Diese Variabilität in der Zusammensetzung der Butterfette macht es dem Nahrungsmittelchemiker, sofern es sich nicht um grobe Fälschungen handelt, um so schwerer, einen Zusatz von Fremdfett in Butter zu erkennen, als die zu Gebote stehenden Verfahren in der Regel auf "quantitativen" Reaktionen aufgebaut sind.

So mißlich es erscheint, für ein in seiner Zusammensetzung innerhalb so weiter Grenzen schwankendes Naturerzeugnis, wie es die Butter darstellt, analytische Grenzzahlen aufzustellen, so wird man doch nicht darauf verzichten können.

In Übereinstimmung mit dem Verfahren von Polenske und anderen Methoden ist auch unser Verfahren zum Nachweis von Cocosfett in Butter auf dem Unterschiede in der Menge der Glyceride der unlöslichen flüchtigen Fettsäuren im Cocosfett einerseits, im Butterfett andererseits aufgebaut und die Aufstellung von Grenzzahlen bleibt auch hier eine gegebene Notwendigkeit. Dies ergibt sich aus der Betrachtung der Tabelle X (S. 46-51), in welcher sämtliche untersuchten Proben, nach steigenden Verseifungszahlen geordnet, zusammengestellt sind und aus welcher eine Zunahme in der Höhe der Cadmium-Zahlen mit ansteigenden Verseifungszahlen unverkennbar ist, während der Zusammenhang der Reichert-MeiBl'schen Zahl mit der Cadmiumzahl weniger ausgeprägt erscheint (vergl. Cocoskuchenfütterung in Tabelle V). Zwar korrespondieren in manchen Fällen die Reichert-Meiß 'schen Zahlen auch mit den Cadmium-Zahlen, aber man findet auch eine Anzahl von Butterfetten mit ähnlichen Reichert-M eifl'schen Zahlen, denen recht verschiedene Cadmium-Zahlen gegenüberstehen. Diese Abweichungen treten, wie aus Tabelle $\mathrm{X}$ ersichtlich ist, zugleich mit mehr oder minder hohen, negativen Jucken a ck-Pastern ack'schen Differenzen auf, die ja nichts anderes besagen, als daß in derartigen Milchfetten die Menge der Glyceride der flüchtigen unlöslichen Säuren gegenüber der der flüchtigen wasserlöslichen Säuren über die Norm erhöht ist. Då der Tierkörper solche Milchfette erzeugt, steht auf Grund der Arbeiten anderer Forscher, wie auch nach dem Ergebnis vorliegender Untersuchung fest. Darum kann auch die Methode von Polenske, einer bestimmten Reichert-Meißl'schen Zahl immer die gleiche oder nur innerhalb geringer Grenzen schwankende „neue Butterzahl“ entgegenzusetzen, zu Irrtümern führen.

Jedenfalls können die Cadmium-Zahlen in kein direktes Abhängigkeitsverhältnis zu den Reichert-Meißl'schen Zahlen gebracht werden.

Aus Tabelle $\mathrm{X}$ ergibt sich, daß die Cadmium-Zahlen von weitaus den meisten Butterproben zwischen den Werten 70-90 liegen. Cadmium-Zahlen zwischen 70-75 werden hauptsächlich bei Milchfetten von altmilchenden Kühen erhalten, oder auch unter dem Einflusse proteinreicher Ernährung (Malzkeime). Die Wirkung der Cocoskuchen- und Rübenfütterung auf die Verminderung der Glyceride der flüchtigen, löslichen Säuren bezw. auf die Vermehrung der Glyceride der flüchtigen unlöslichen

[Fortsolzung S. 50]. 
Tabelle $\mathrm{X}$.

\begin{tabular}{|c|c|c|c|}
\hline No. & Herkunft der Butter & Fütterungsart & $\begin{array}{l}\text { Zeit der Proben- } \\
\text { entnahme }\end{array}$ \\
\hline 1 & Gut I, Altdorf bei Nürnberg . . . & Malzkeime & April 1907 \\
\hline \begin{tabular}{c|}
2 \\
3 \\
4 \\
5 \\
6 \\
7 \\
8 \\
9 \\
10 \\
$\left.11^{1}\right)$ \\
12
\end{tabular} & Gut II, Altdorf bei Nürnberg & $\begin{array}{c}\text { Heu, Gras } \\
\text { Heu, Häcksel, 15 Pfd. Rüben } \\
\text { Heu, Gras, Häcksel } \\
\text { Heu, Gras, Rüben } \\
\text { Hen, Häcksel, Treber } \\
\text { Heu, Häcksel. 2 Pfd. Cocoskuchen } \\
\text { Gras, Häcksel } \\
\text { Heu, Häcksel, } 20 \text { Pfd. Rüben } \\
\text { Heu, Gras, 1,5 Pfd. Cocoskuchen } \\
\text { Heu, Gras } \\
\text { Heu, Gras, 2 Pfd. Cocoskuchen }\end{array}$ & $\begin{array}{c}\text { April } 1907 \\
\text { Oktober } 1907 \\
\text { Oktober } 1907 \\
\text { Oktober } 1907 \\
\text { Mitte Oktober } \\
1907 \\
\text { Oktober } 1907 \\
\text { Oktober } 1907 \\
\text { Oktober } 1907 \\
\text { Oktober } 1907 \\
\text { Anfang Oktober } \\
\text { 1907 } \\
\text { Oktober } 1907\end{array}$ \\
\hline 13 & Braunan (Ober-Österreich) . . . . & unbekannt, Sammel-Butter & Oktober 1907 \\
\hline $\begin{array}{l}14 \\
15 \\
16\end{array}$ & Gut II, Altdorf bei Nürnberg & $\begin{array}{l}\text { Heu, Hăcksel, } 25 \text { Pfd. Rüben } \\
\text { Hea, Gras, } 4 \text { Pfd. Cocoskuchen } \\
\text { Heu, Gras, 1,5 Pfd. Cocoskuchen }\end{array}$ & $\begin{array}{c}\text { Oktober } 1907 \\
\text { Oktober } 1907 \\
\text { Juli } 1907\end{array}$ \\
\hline 17 & Molkerei Gobsan (Brandenburg) . . & Grünfutter, Rüben, Schlempe & November 1906 \\
\hline 18 & Molkerei Sagast (Brandenburg) & Klee, Heu, Haferstroh, Weidegang & November 1906 \\
\hline 191) & Tennenlohe kei Erlangen . . . & unbekannt & Мai 1907 \\
\hline$\overline{20}$ & Molkerei Großenbuch (Oberfranken) & Heu, Gras & Mai 1907 \\
\hline 21 & Gut II, Altdorf bei Nürnberg. . & 24 Pfd. Treber & März 1907 \\
\hline 22 & $\begin{array}{l}\text { Radstädter Tquern (Salzburg), Hoch- } \\
\text { alpe, } 1000 \mathrm{~m}\end{array}$ & Weidegang & September 1906 \\
\hline 23 & Gut II, Altdorf bei Nürnberg. . . & Gras, Häcksel & Juli 1907 \\
\hline 24 & Gräfenberg (Oberfranken) . . . & unbekannt & Mai 1907 \\
\hline 25 & Gat II; Altdorf bei Nürnberg & Weidegang & Juli 1907 \\
\hline 26 & Fichtelberg (Oberfranken) . . . . & unbekannt & Oktober 1907 \\
\hline 27 & Windsbach (Mittelfranken). . . . & Weidegang & September 1906 \\
\hline 28 & Reichelsdorf bei Nürnberg . . . . & Weidegang & Oktober 1906 \\
\hline
\end{tabular}

1) Bei größeren Cocosfettzusătzen ergaben sich bei den Butterfetten Nr. 11 und 19 folgende Cadminm-Zahlen:

$\begin{array}{cccc}\text { Butterfett } & 15 \% \text { Cocosfett } & 20 \% \text { Cocosfett } & 30 \% \text { Cocosfett } \\ \text { No. } 11 & 121,0 & 150,0 & 179,0 \mathrm{mg} \\ \text { No. } 19 & 129,0 & 167,4 & 183,4 \mathrm{mg}\end{array}$


17. Band. Januar 1909.] Pa a I u. A mberger, Nachweis von Cocosfett in Butterfett.
.

Übersichtstabelle.

\begin{tabular}{|c|c|c|c|c|c|c|c|c|}
\hline \multirow{2}{*}{$\begin{array}{c}\text { Ver- } \\
\text { seifungs- } \\
\text { zahl }\end{array}$} & \multirow{2}{*}{$\begin{array}{l}\text { Reichext- } \\
\text { Meisl'sche } \\
\text { Zahl }\end{array}$} & \multirow{2}{*}{$\begin{array}{l}\text { Reichert- } \\
\text { Meifl'sehe } \\
\text { Zahl nach } \\
\text { Polenske } \\
\text { bestimmt }\end{array}$} & \multicolumn{2}{|c|}{ Polenste'sche Zahl } & \multirow{2}{*}{$\begin{array}{c}\text { Jucke- } \\
\text { nack-Pa- } \\
\text { sternacks:- } \\
\text { sche } \\
\text { Differenz }\end{array}$} & \multicolumn{2}{|c|}{ Oadminm-Zabl } & \multirow{2}{*}{ Bemerikungen } \\
\hline & & & gofunden & normal & & Butterfett & $\begin{array}{l}\text { Butterfett } \\
\text { mit } 10 \% \\
\text { Cocosfett }\end{array}$ & \\
\hline 218,0 & 18,0 & - & - & - & \pm 0 & 70,0 & 104,0 & - \\
\hline 218,2 & 20,3 & 20,6 & 1,2 & $1,35-1,4$ & $+2,1$ & 72,0 & - & (Tab. V, 9) \\
\hline 219,2 & 22,4 & - & - & - & $+3,2$ & 71,0 & 106,0 & - \\
\hline 220,0 & 22,3 & 22,5 & 1,4 & 1,5 & $+2,3$ & 76,4 & 112,2 & (Tab. VIII, 1) \\
\hline 220,5 & 22,7 & 22,4 & 1,35 & 1,5 & $+2,2$ & 71,0 & 104,3 & (Tab. VIII, 2) \\
\hline 220,6 & 23,1 & 23,0 & 1,6 & 1,6 & $+2,5$ & 69,8 & 102,0 & $\begin{array}{l}\text { (Tab. IX, 4) } \\
\text { altmilehend }\end{array}$ \\
\hline 221,0 & 22,7 & 22,1 & 1,7 & $1,4-1,5$ & $+1,7$ & 76,4 & 112,2 & (Tab. V, 2) \\
\hline 221,4 & 22,3 & 22,0 & 1,45 & $1,4-1,5$ & $+0,9$ & 88,0 & 114,0 & (Tab.V,Vorp.) \\
\hline 221,8 & 28,1 & 23,4 & 1,6 & 1,5 & $+1,3$ & 79,8 & 114,6 & (Tab. VIII, 7) \\
\hline 221,9 & 28,5 & 23,3 & 1,5 & $1,5-1,7$ & $+1,6$ & 74,0 & 116,0 & (Tab. $\nabla, 1$ ) \\
\hline 222,7 & 23,3 & 23,5 & 1,7 & $1,5-1,7$ & $+0,6$ & 70,4 & $\left.108,0^{1}\right)$ & $\begin{array}{l}\text { Tab. III,I u. IX, } \\
\text { 3)altmilchend }\end{array}$ \\
\hline 222,8 & 22,0 & 21,7 & 2,05 & $1,4-1,5$ & $-0,8$ & 88,8 & - & - \\
\hline 223,1 & 24,9 & - & - & - & $+1,8$ & 890 & 117,0 & - \\
\hline 223,6 & 24,7 & 24,9 & 1,95 & 1,7 & $+1,1$ & 77 & 110,0 & Tab. VIII, 3) \\
\hline 223,8 & 19,6 & 19,6 & 2,15 & 1,35 & $-4,2$ & 87,0 & - & $(\mathrm{Tab} . \mathrm{V}, 4)$ \\
\hline 223,8 & 25,1 & - & - & 一 & $-1,3$ & 81,4 & 114,0 & - \\
\hline 224,0 & 27,1 & $=$ & - & - & $+3,1$ & 78,8 & 116,6 & - \\
\hline 224,0 & 28,2 & - & - & - & $+4,2$ & 79,6 & 114,0 & - \\
\hline 224,2 & 22,2 & 22,4 & 1,6 & 1,5 & $-2,0$ & 82,0 & $\left.116,0^{1}\right)$ & $\begin{array}{l}\text { Tab, II, a. IX, } \\
\text { 5) } 28-30 \text { Wo- } \\
\text { chen trächtig }\end{array}$ \\
\hline 224,4 & 24,2 & - & - & - & $-0,2$ & 82,0 & 119,0 & - \\
\hline 224,4 & 26,2 & 26,3 & 2,3 & 1,9 & $+1,8$ & 88,0 & 122,6 & - \\
\hline 224,5 & 22,4 & - & - & - & $-2,1$ & 86,4 & 114,4 & - \\
\hline 224,9 & 24,2 & 24,6 & 1,9 & $1,6-1,7$ & $-0,7$ & 81,2 & - & (Tab. IX, 2) \\
\hline 225,3 & 25,6 & - & - & - & $+0,3$ & 78,0 & 115,0 & - \\
\hline 226,5 & 25,6 & 25,6 & 1,9 & 1,7 & $-0,9$ & 89,0 & 116,0 & - \\
\hline 226.5 & 28,6 & - & - & - & $+2,1$ & 79,0 & 112,0 & - \\
\hline 227,0 & 24,0 & - & - & - & $-3,0$ & 84,0 & 118,0 & - \\
\hline 227,2 & 27,7 & - & - & - & $+0,5$ & 84,0 & 119,8 & - \\
\hline
\end{tabular}


Tabelle X.

\begin{tabular}{|c|c|c|c|}
\hline No. & Herkunft der Butter & Fütterungsart & $\begin{array}{c}\text { Zeit der } \\
\text { Probenentnabme }\end{array}$ \\
\hline 29 & Gut II, Altdorf bei Nürnberg. . . & 30 Pfd. Rüben & Oktober 1907 \\
\hline 30 & $\begin{array}{c}\text { Radstädter Tauern (Salzburg), Gut I, } \\
1000 \mathrm{~m} \text { hoch gelegen }\end{array}$ & Hen, Wruken & April 1907 \\
\hline 31 & Gut II, Altdorf bei Nürnberg. . . & 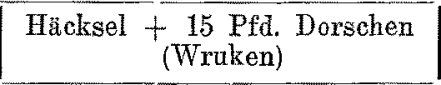 & Februar 1907 \\
\hline 32 & Braunau (Ober-Österreich) . . . . & unbekannt, Sammelbutter & Oktober 1906 \\
\hline 33 & Grokenbuch (Oberfranken). : & Weidegang & Oktober 1906 \\
\hline 34 & Gut II, Altdorf bei Nürnberg. . . & 40 Pfd. Rüben & Oktober 1907 \\
\hline 35 & Kochstedt bei Dessau $-\ldots$. & unbekannt & Januar 1907 \\
\hline 36 & Gut II, Altdorf bei Nürnberg. . . & Heu, Häcksel, 6 Pfd. Cocoskuchen & Oktober 1907 \\
\hline 37 & Molkerei Schwiebus (Brandenburg). & unbekannt & November 1906 \\
\hline 38 & Gut II, Altdorf bei Nürnberg. . . & 50 Pfd Rüben & Oktober 1907 \\
\hline 39 & Gut II, Altdorf bei Nürnberg. . & Häcksel +15 Pfd. Dorschen & Februar 1907 \\
\hline 40 & Burglengenfeld (Oberpfalz). . . & $\begin{array}{c}\text { Trockenfutter, Rüben, Cocos- } \\
\text { kuchen }\end{array}$ & April 1907 \\
\hline 41 & $\begin{array}{c}\text { Radstädter Tauern (Salzburg), Gut I, } \\
\text { Kuh J. }\end{array}$ & Heu, Gras & Mai 1907 \\
\hline 42 & Gut II, Altdorf bei Nürnberg. . & 7 Pfd. Cocoskuchen & Oktober 1907 \\
\hline 43 & Molkerei Bruck (Brandenburg) & $\begin{array}{c}\text { Heu, Grünfatter, Rübenblätter, } \\
\text { Cocoskuchen }\end{array}$ & Dezember 1906 \\
\hline $\left.44^{x}\right)$ & $\begin{array}{c}\text { Radstädter Tauern, Salzburg, Gut II. } \\
\text { Kuh S. }\end{array}$ & Heu & April 1907 \\
\hline 45 & Kleatsch bei Dessau . . . . . . & Rüben, Kartoffeln & Oktober 1906 \\
\hline $\begin{array}{l}46 \\
47 \\
48\end{array}$ & Gut II, Altdorf bei Nürnberg & $\begin{array}{l}\text { Häcksel }+15 \text { Pfd. Rubben } \\
8 \text { Pfd. Cocoskuchen } \\
10 \text { Pfd. Cocoskuchen }\end{array}$ & $\begin{array}{l}\text { Februar } 1907 \\
\text { Oktober } 1907 \\
\text { Oktober } 1907\end{array}$ \\
\hline 49 & Molkerei Königsberg (Brandenburg). & Wiesen- u. Kleeheu, Schlempe, & Oktober 1906 \\
\hline 50 & Gut Il, Altdorf bei Nürnberg. & Häcksel +60 Pfd. Rüben & Februar 1907 \\
\hline 51 & Molkerei Segrehna (Anhalt) & Heu, Rüben & $\mid$ November 1906 \\
\hline 52 & Molkerei Lausigk (Anhalt). . . . & Zuckerriben-Abfälle & November 1906 \\
\hline
\end{tabular}

1) Bei größeren Cocosfettzusätzen ergaben sich bei dem Butterfett Nr. 44 folgende Cadminm-Zahlen:

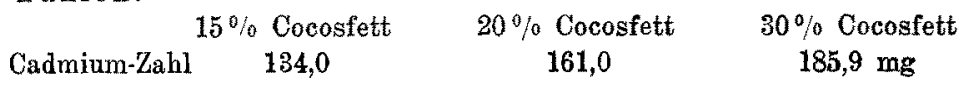


Fortsetzung.

\begin{tabular}{|c|c|c|c|c|c|c|c|c|}
\hline \multirow{2}{*}{$\begin{array}{c}\text { Ver- } \\
\text { seifungs- } \\
\text { zahl }\end{array}$} & \multirow{2}{*}{$\begin{array}{l}\text { Reichert- } \\
\text { Meisl'sche } \\
\text { Zabl }\end{array}$} & \multirow{2}{*}{$\begin{array}{l}\text { Roiehert } \\
\text { Meirl'sche } \\
\text { Zahl nach } \\
\text { Polenske } \\
\text { bestimmet }\end{array}$} & \multicolumn{2}{|c|}{ Polenske'sehe Zabl } & \multirow{2}{*}{\begin{tabular}{|c|} 
Jucke- \\
nack-Pa- \\
sternac \\
sche \\
Differenz
\end{tabular}} & \multicolumn{2}{|c|}{$\mathrm{Cadmium-Zahl}$} & \multirow[b]{2}{*}{ Bemerkungen } \\
\hline & & & gefunden & normal & & Butterfett & $\begin{array}{l}\text { Butterfett } \\
\text { mit } 10 \% \\
\text { Oocosfett }\end{array}$ & \\
\hline 227,6 & 25,5 & 25,5 & 2,05 & 1,9 & $-2,1$ & 81,0 & 116,0 & (Tab. VIII, 4) \\
\hline 227,7 & 29,0 & - & - & - & $+1,3$ & 87,4 & 122,6 & - \\
\hline 227,8 & 25,8 & 26,0 & 1,9 & 1,9 & $-2,0$ & 96,0 & - & (Tab. VII, 2) \\
\hline 228,1 & 26,6 & - & - & - & $-1,5$ & 86,0 & 124,0 & - \\
\hline 228,3 & 27,5 & - & - & - & $-0,8$ & 81,6 & 118,4 & 一 \\
\hline 228,6 & 25,9 & 26,1 & 2,5 & 1,9 & $-2,7$ & 86,0 & 124,0 & (Tab. VIII, 5) \\
\hline 228,6 & 27,7 & - & - & - & $-0,9$ & 80,0 & 115,0 & - \\
\hline 229,0 & 21,9 & 22,0 & 2,3 & $1,4-1,5$ & $-7,1$ & 94,0 & - & (Tab. V, 5) \\
\hline 229,0 & 26,6 & - & - & - & $-2,4$ & 77,0 & 114,0 & 一 \\
\hline 229,1 & 26,7 & 26,5 & 2,6 & 1,9 & $-2,4$ & 88,7 & 118,3 & (Tab. VIII, 6) \\
\hline 229,6 & 26,4 & 26,5 & 1,8 & 1,9 & $-3,2$ & 86,0 & - & (Tab. VII, 8) \\
\hline 229,6 & 22,2 & 22,6 & 2,1 & 1,5 & $-7,4$ & 90,0 & 114,0 & (Tab. VI, 1) \\
\hline 229,8 & 31,0 & - & 一 & - & $+1,2$ & 88,0 & 121,2 & - \\
\hline 230,2 & 21,5 & 21,46 & 2,45 & $1,4-1,5$ & $-8,7$ & 106,0 & - & (Tab. V, 6) \\
\hline 230,5 & 28,4 & - & - & - & $-2,1$ & 90,0 & 122,0 & - \\
\hline 230,6 & 32,3 & - & 一 & - & $+1,7$ & 90,0 & $\left.121,4^{1}\right)$ & (Tab. III, 3) \\
\hline 231,3 & 29,0 & - & - & - & $-2,3$ & 89,0 & 124,0 & - \\
\hline 232,3 & 27,0 & 27,17 & 2,3 & 1,9 & $-5,3$ & 92,0 & - & (Tab. VII, 7) \\
\hline 232,4 & 19,9 & 19,85 & 2,7 & 1,35 & $-12,5$ & 108,0 & 一 & $(\mathrm{Tab} . \mathrm{V}, \mathrm{7})$ \\
\hline 232,7 & 19,8 & 19,65 & 2,7 & 1,35 & $-12,9$ & 112,0 & - & $(\mathrm{Tab} . \mathrm{V}, 8)$ \\
\hline 233,2 & 27,1 & 27,35 & 2,3 & 1,9 & $-6,1$ & 88,0 & 117,0 & (Tab. VI, 2) \\
\hline 233,2 & 27,9 & 27,98 & 2,9 & $2,2-2,3$ & $-5,3$ & 106,9 & - & (Tab. VII, 3) \\
\hline 234,3 & 28,2 & 28,3 & 2,7 & 2,3 & $-6,1$ & 108,0 & - & (Tab. VI, 4) \\
\hline 235,2 & 30,1 & 29,92 & 3,7 & $2,5-3$ & $-5,1$ & 111,0 & 144,0 & (Tab. VI, 7) \\
\hline
\end{tabular}


Tabelle X.

\begin{tabular}{|c|c|c|c|}
\hline No. & Herkunft der Butter & Fütterungsart & $\begin{array}{l}\text { Zeit der Proben- } \\
\text { entnahme }\end{array}$ \\
\hline 53 & Gat II, Altdorf bei Nürnberg. . . & Häcksel +45 Pfd. Räben & Februar 1907 \\
\hline 54 & Molkerei Dessan (Anhalt). . . . & Zuckerrüben-Abfälle & November 1906 \\
\hline 55 & Kochstedt (Anhalt). & Rüben, Rübenkraut, Cocoskuchen & November 1906 \\
\hline 56 & Gut II, Altdorf bei Nürnberg. . . & Häcksel +75 Pfd. Rüben & Februar 1907 \\
\hline 57 & Quellendorf (Anhalt) . . . . . . & Zuckerrüben-Abfalle & November 1906 \\
\hline 58 & Gat II, Altdorf bei Nürnberg. . . & Häcksel +90 Pfd. Rüben & Februar 1907 \\
\hline 59 & Gut II, Altdorf bei Nürnberg. . . & $\begin{array}{c}75 \text { Pfd. Runkelrüben, } 16,5 \text { Pfd. } \\
\text { Dorschen, } 3 \text { Pfd. Malzkeime }\end{array}$ & Februar 1907 \\
\hline 60 & Gut I, Altdorf bei Nürnberg & Rüben & November 1907 \\
\hline
\end{tabular}

[Fortsetzung von S. 45.]

Säuren und damit auf die Cadmium-Zahl macht sich erst bei großen Gaben dieser Futtermittel bemerkbar. Die Cadmium-Zahlen erreichen dann den Wert 100 und darüber. Auch zeigt sich, daß die Polenske'sche Zahl im Vergleich zur Cadmium-Zahl bei Butter von mit Rüben oder Cocosfett gefütterten Kühen rascher über die Norm ansteigt, wie die Cadmium-Zahl. (Vergl. Tabelle X, No. 7, 12, 15, 21, $34,36,38,40,4649$.)

Während nun bei starker Rübenfütterung Reichert-Meiß I'sche Zahlen und Verseifungszahlen ansteigen, letztere aber in höherem Maße wie erstere, zeigt sich bei Cocosfütterung das Umgekehrte; die Reichert-Meißl'sche Zahl sinkt, während die Verseifungszahl steigt, letztere jedoch nicht so boch, wie bei Rübenfütterung. In beiden Fällen müssen sich natürlich hohe negative Juckenack-Pasternack'sche Differenzen'ergeben. Nicht uninteressant ist das Verhältnis dieser Werte bei ge mischter Cocosfett-Rübenfütterung. Die Reichert-MeiBl'schen Zahlen liegen dann zwischen den niedrigen Werten bei reiner Cocosfütterung und den hohen bei ausschließlicher Rübenfütterung. Die Verseifungszahlen werden unter dem Einfluß beider Futtermittel erhöht, aber nicht in dem Maße, wie bei starker Rübenfütterung allein (vergl. Tab. $\mathrm{X}$ No. 40, 43, 55).

Vergleicht man die Cadmium-Zahlen der reinen Butterfette mit denen einer Mischung von Butter mit 20\% Cocosfett, so findet man, daß die Cadmiumzahlen dieser Mischungen weit über den höchsten, bei reinen Butterfetten erhaltenen Werten liegen. Derartige Verfälschungen werden also auf Grund der Cadmium-Zahlen ohne weiteres erkennbar sein, vorausgesetzt, daß nicht in Zukunft noch böhere Cadmiumzahlen für reine Butterfette gefunden werden. Anders liegen die Verhältnisse, wenn es sich um den Nachweis geringerer Mengen Cocosfett in Butterfett handelt. In solchen Fällen wird man nur unter gleichzeitiger Berücksichtigung der Reichert-Meißl' schen Zahl und der Verseifungszahl sowie der Juckenack-Pasternack'schen Differenz Anhaltspunkte dafür erhalten, ob ein Butterfett kleine Mengen Cocosfett $(10-15 \%)$ enthält oder nicht. 
Fortsetzung.

\begin{tabular}{|c|c|c|c|c|c|c|c|c|}
\hline \multirow{2}{*}{$\begin{array}{c}\text { Ver- } \\
\text { seifungs } \\
\text { zahl }\end{array}$} & \multirow{2}{*}{$\begin{array}{c}\text { Reichert- } \\
\text { Meis I'sche } \\
\text { Zahl }\end{array}$} & \multirow{2}{*}{$\begin{array}{l}\text { Reiohert- } \\
\text { Moifl 'sche } \\
\text { Zabl nach } \\
\text { Polenske } \\
\text { bestimmt }\end{array}$} & \multicolumn{2}{|c|}{ Polens ke'sehe Zahl } & \multirow{2}{*}{$\begin{array}{c}\text { Jucke- } \\
\text { naek-Pa- } \\
\text { sternatk'- } \\
\text { sche } \\
\text { Differenz }\end{array}$} & \multicolumn{2}{|c|}{ Cadminm-Zahl } & \multirow[b]{2}{*}{ Bemerkungen } \\
\hline & & & gefumden & normal & & Butterfett & $\begin{array}{l}\text { Butterfott } \\
\text { mit } 10 \% \\
\text { Cocosfett }\end{array}$ & \\
\hline 236,9 & 28,6 & 28,9 & 2,8 & 2,5 & $-7,7$ & 112,0 & - & $(\mathrm{Tab}, \mathrm{VII}, 6)$ \\
\hline 236,5 & 30,4 & 29,86 & 3,5 & $2,5-3$ & $-6,1$ & 117,0 & 146,0 & (Tab. VI, 6) \\
\hline 236,5 & 27,1 & 27,35 & 2,8 & 1,9 & $-9,4$ & 112,0 & 141,0 & (Tab. VI, 3) \\
\hline 237,0 & 29,4 & 29.5 & 3,4 & $2,6-2,7$ & $-7,6$ & 112,0 & 一 & (Tab. VII, 4) \\
\hline 237,3 & 29,3 & 29,54 & 3,4 & $2,6-2,7$ & $-8,0$ & 122,0 & 1054,0 & (Tab. VI, 5) \\
\hline 238,7 & 30,0 & 29,92 & 8,6 & $2,6-3$ & $-8,7$ & 117,0 & 一 & $(\mathrm{Tab}$ VII, 5) \\
\hline 238,9 & 31,0 & 31,15 & 3,6 & 3 & $-7,9$ & 130,0 & - & $(\mathrm{Tab} . \mathrm{VII}, 1)$ \\
\hline 248,1 & 31,1 & 31,2 & 3,8 & - & $-12,0$ & 129,6 & - & $(\mathrm{Tab}, \mathrm{VT}, 8)$ \\
\hline
\end{tabular}

Von diesen Gesichtspunkten ausgehend, schlagen wir vorläufig und mit allem Vorbehalt folgenden Entwurf zur Aufstellung von Grenzzahlen vor:

Butter ist als mit Cocosfett vermischt zu beanstanden:

1. Wenn die Cadmium-Zahl über 100 liegt und die Juckenack-Pastern ack'sche Differenz sich innerhalb der Grenzwerte $+4,25$ bis $-3,5$ bewegt.

2. Wenn bei einer Verseifungszahl von höchstens 235 und einer JuckenackPasternack'schen Differenz von böchstens bis $\mathrm{zu}-8$ die Cadmium-Zahl den Wert 110 übersteigt.

3. Wenn bei einer Verseifungszahl von höchstens 235 und einer Jucken ack Pasternack'schen Differenz von mehrals - 8die Cadınium-Zahl über 120 liegt.

Aufierhalb dieses Satzes würden nur die Proben Nr. 40 and 49 der Tabelle $X$ fallen. welche bei einem Zusatz von 10\% Cocosfett die unter 120 liegenden Cadmium-Zahlen 114 und 117 ergaben. Ein Zusatz von $15 \%$ Cocosfett würde auch in diesen Fällen nachweisbar sein.

4. Wenn bei einer Verseifungszahl von über 235 die Cadmium-Zahl den Wert 130 übersteigt.

5. SchlieBlich läßt sich mit Rücksicht auf die Praxis sagen, daß Butter, deren Reichert-Meibl'sche Zahl 28 und darüber beträgt, nicht mehr beanstandet werden sollte.

Wenn wir auch bemüht waren, uns ein mannigfaltiges Material an Butterproben zu verschaffen, so sind wir uus doch bewußt, dal es noch viel zu gering ist, um über die Brauchbarkeit des von uns vorgeschlagenen Verfahrens entscheiden zu können. Wir bitten daher die verehrten Herren Fachgenossen, die Methode nach Möglichkeit nachprüfen zu wollen.

Wir erfüllen zum Schlusse eine angenehme Pflicht, wenn wir den Herren Dr. Leuze-Dessau, Dr. W. Fuchs und Dr. Zitelmann-Berlin für die Übermittelung reiner Butter- und Rahmproben, sowie Herrn Landwirtschaftslehrer Weidringer in Altdorf für die Beaufsichtigung der Fütterungsversuche auch an dieser Stelle unsern verbindlichsten Dank sagen. 